\title{
Activités
}

8-1 | avril 2011

Varia

\section{Isolement, parcellisation du travail et qualité des soins en gériatrie}

Isolation, fragmentation of work and quality of care in geriatrics

Frédéric Garcia, Deslandes Hélène, Anne-Emilie Ravache, Thierry Bitot, Elise Chaumon, Giuliana Milanini-Magny et Madeleine Estryn-Behar

\section{OpenEdition}

1 Journals

Édition électronique

URL : http://journals.openedition.org/activites/2516

DOI : $10.4000 /$ activites. 2516

ISSN : 1765-2723

Éditeur

ARPACT - Association Recherches et Pratiques sur les ACTivités

Référence électronique

Frédéric Garcia, Deslandes Hélène, Anne-Emilie Ravache, Thierry Bitot, Elise Chaumon, Giuliana Milanini-Magny et Madeleine Estryn-Behar, «Isolement, parcellisation du travail et qualité des soins en gériatrie », Activités [En ligne], 8-1 | avril 2011, mis en ligne le 15 avril 2011, consulté le 30 avril 2019. URL : http://journals.openedition.org/activites/2516 ; DOI : 10.4000/activites.2516

\section{(c) (i) (2)}

Activités est mis à disposition selon les termes de la licence Creative Commons Attribution - Pas d'Utilisation Commerciale - Pas de Modification 4.0 International. 


\title{
Isolement, parcellisation du travail et qualité des soins en gériatrie
}

\author{
Madeleine Estryn-Behar \\ madeleine.estryn-behar@sap.aphp.fr \\ Elise Chaumon \\ ergonome, Hôtel Dieu, Paris. \\ elise chaumon elise.chaumon@ @otmail.fr \\ Frédéric Garcia \\ ergonome, Hôtel Dieu, Paris. \\ f2.garcia@laposte.net
}

médecin du travail épidémiologiste ergonome, Hôtel Dieu Parvis Notre Dame 75004 Paris France.

\author{
Giuliana Milanini-Magny \\ architecte, Hôtel Dieu, Paris. \\ giuliana_magny@numericable.fr' \\ Thierry Bitot \\ médecin du travail, ergonome, $\mathrm{CH}$ de Montbrison \\ thierrybitot@yahoo.fr
}

\author{
Anne-Emilie Ravache \\ ergonome, Hôtel Dieu, Paris. \\ anne-emilie.ravache@wanadoo.fr
}

\section{Deslandes Hélène \\ ergonome, Hôtel Dieu, Paris. \\ deslandeshelene@yahoo.fr}

\begin{abstract}
Isolation, fragmentation of work and quality of care in geriatrics. The organisation of work and space have a major impact on how health workers do their job. The core of their occupation is to provide basic care for residents of nursing homes and patients in geriatric departments who are often very dependent. This participatory ergonomics analysis set out to understand why prescribed work does not always tally with actual work, with a view to suggesting improvements that will benefit both health workers and patients.
\end{abstract}

A real-time task analysis of 40 full days worked by nursing assistants (NAs, $\mathrm{n}=26$ ) and registered nurses (RNs, $\mathrm{n}=14$ ) was carried out by ergonomists and health workers taking part in the research.

In theory, the geriatric nurses and nursing assistants should be supporting and stimulating patients, often at the end of their lives, and giving them appropriate prescribed treatment. The real-time task analysis showed that they could only stay with patients for short periods (for NAs, 68 periods and $44 \%$ of their working time; for RNs, 51 periods and $22 \%$ of their working time). NAs spend very little time at the nurses' workstation $(5.9 \%$ compared to $41.5 \%$ for RNs) which means they have few opportunities for discussion with RNs. On average, NAs spend only $1.8 \%$ of their working time in discussion with RNs, even though it is the NAs who spend the most time talking to patients. Little use seems to be made of their observations in adapting the "life projects" of patients - in contradiction with the certification criteria.

Moreover, they seldom have time to share their emotional burden with their colleagues. 
Because of the way health workers' tasks are organised, they devote a large amount of time to indirect care such as preparation and cleaning. For the RNs, this means administrative tasks and walking from one ward to another. As a result, there is a danger of loneliness and a high risk of professional burnout.

This method suggests improvements to working conditions, cooperation between the occupations concerned and the quality of patient care, as part of a multidisciplinary group.

KEYWORDS

Participatory ergonomics, geriatric care, burnout, emotional sharing, quality of care

\section{1.- Introduction}

La thématique de la souffrance au travail est de plus en plus prégnante, et ce qu'elle soit physique ou mentale (Dejours, 1998; Carré, 2007). De multiples facteurs sont à l'origine de cette problématique. Ils peuvent être d'ordres environnementaux, organisationnels, architecturaux, sociaux, économiques, etc.

L'isolement a été montré comme un des plus sûrs précurseurs des décompensations psychologiques (Davezies, 2007). Les liens entre absence de support social, forte charge de travail, manque d'autonomie, et épuisement professionnel, ont été mis en évidence en France dans différentes branches professionnelles (Huez, 2008). Cette étude, reprenant les modèles classiques de Karasek (1990) et Siegrist (1996), a aussi souligné la relation entre le déséquilibre effort / récompense et de nombreux problèmes de santé.

Au sein des secteurs hospitaliers, sanitaires et sociaux, les soignants peuvent être soumis à plusieurs de ces contraintes, engendrant davantage de difficultés dans l'exercice de leur profession (EstrynBehar, 1997).

L'étude statistique longitudinale PRESST-NEXT, menée dans une partie des établissements étudiés lors des interventions ergonomiques présentées ici, a montré le lien entre qualité du travail d'équipe et arrêts maladies, mais aussi burnout et départ prématuré de la profession. Au cours de deux enquêtes réalisées à un an d'intervalle, sur un échantillon européen de près de 40000 soignants paramédicaux, il a été montré que le facteur ayant le plus d'influence sur l'intention d'abandonner la profession est la faiblesse du travail d'équipe. Cette intention d'abandon est multipliée par six dans l'enquête initiale, et par cinq un an après, chez les soignants dont le travail d'équipe est considéré comme insuffisant par rapport à ceux qui ont une bonne qualité de ce travail d'équipe. L'épuisement professionnel ou «burnout » double l'intention d'abandon dans les deux enquêtes et son influence s'accentue au cours de l'étude (Odds ratio ajusté $($ ORaj) = 2,19 en 2003 et ORaj = 2,30 en 2004). L'influence des conflits entre travail et vie personnelle dans l'intention de départ s'accroît (ORaj = 1,44 en 2003 et ORaj = 1,69 en 2004) et les faibles possibilités de développement professionnel augmentent d'une fois et demie l'intention d'abandonner. Les troubles de la santé mentale renforcent, eux aussi, d'une fois et demie l'intention d'abandonner, alors que les troubles musculo-squelettiques (TMS) ont une influence minime parmi les répondants au questionnaire initial et aucune parmi ceux ayant répondu un an plus tard. En effet, les troubles de la santé mentale n'invalident pas la possibilité de trouver un emploi ailleurs contrairement aux TMS, ce qui a pu avoir un impact sur les réponses apportées au questionnaire. Par ailleurs cette étude a montré que parmi les 5376 soignants étudiés en France, ceux travaillant en hôpitaux locaux dans des services de long séjour gériatriques et ceux travaillant en maison de retraite étaient les plus insatisfaits de leurs possibilités de donner à leurs patients la qualité des soins dont ils ont besoin et qu'ils n'avaient majoritairement pas le temps suffisant pour parler aux malades ou résidents (Estryn-Behar, Le Nézet, Loriol, Ben-Brik, \& Caillard, 2003 ; Estryn-Behar, 
Salbreux, Paoli, \& Le Nezet, 2007; Estryn-Behr, Van der Heijden, Oginska, Camerino, Le Nezet, Conway, et al., 2007). La mise en place des 35 heures, réduction du temps de travail (RTT) s'est le plus souvent faite sans augmentation du personnel au lit du malade, initialement, et en réduisant les temps de chevauchement entre équipes successives qui servaient à souder les équipes. La «mutualisation des moyens » et la polyvalence se sont développées. Aussi, si les 5376 soignants français considèrent pour la plupart que cela a eu un effet positif sur leur vie personnelle, ils considèrent majoritairement que leurs conditions de travail sont plus difficiles depuis la RTT.

Les études ergonomiques menées en milieu hospitalier et médico-social ont montré que la parcellisation du travail des soignants, entre la recherche d'information, la traçabilité, la préparation et le rangement des soins et leur réalisation, empêche un accompagnement du patient passant essentiellement par la présence du soignant à ses côtés pour une possibilité de soins relationnels (Estryn-Behar, 1996, 1997, 2001 ; Estryn-Behar et Poinsignon, 1989; Rogez, 2009; Villate, Gadbois, Bourne, \& Viser, 1993). Cette insuffisance de temps disponible pour une relation personnalisée avec des résidents de structures de soins ou d'hébergement gériatrique, a été mise en évidence par des gériatres comme liée à un état dépressif constaté chez certains sujets âgés. Cet état affaiblit la part d'autonomie restante qui peut être travaillée et donc préservée avec l'aide d'un soignant (Bartels, Shea, Beck, Clark, Mansfield, Kpp, \& Ouslander, 2003 ; Stephen, Bartels, Aricca, Dums, Thomas, Oxman, et al., 2004).

Les débats actuels autour du décret de compétence infirmière mettent l'accent sur les soins relation$n e l s$. C'est pourquoi les différences entre travail prescrit et travail réel sont particulièrement génératrices de souffrance au travail parmi les soignants. Les soins infirmiers sont constitués d'échanges dynamiques entre l'infirmier et une personne ou un groupe de personnes. Ils se caractérisent, selon la catégorisation enseignée dans les instituts de formation (Soins Infirmiers Individualisés à la Personne Soignée ou SIIPS), par des soins « de base » (liés à l'alimentation, la locomotion, la mobilisation, l'élimination, l'hygiène et le confort), «techniques » (liés à la prescription médicale, l'aide aux actes diagnostiques, la surveillance des traitements), « éducatifs et relationnels » (de type verbal ou non, permettant une communication dans le but d'aider une personne ou un groupe de personnes, en dehors du commentaire des soins administrés). Tous ces soins requièrent expertise technique, disponibilité, observation, capacité d'analyse, écoute, compréhension des problèmes, respect de la différence, accompagnement, relation d'aide (Daydé, 2008; Daydé, Lacroix, Pascal, \& Salabara Clergues, 2007), permanence et continuité. Ils impliquent la connaissance et l'application de techniques de soins spécifiques somatiques et la maîtrise des concepts relatifs au comportement à la personnalité, au psychisme, aux différentes pathologies somatiques et psychiques et enfin aux relations interpersonnelles.

Les soins infirmiers préventifs, curatifs ou palliatifs sont donc aussi bien de nature technique, que d'aide à la continuité des fonctions de base ou de nature relationnelle et éducative. Leur réalisation prend en compte l'évolution des sciences et des techniques. Le site infirmier Serpsy (www.serpsy. fr) développe les concepts enseignés aux soignants. Ces trois aspects sont indissociables de tout soin infirmier. Ils ont pour objet, dans le respect des règles professionnelles des infirmiers et infirmières, incluant notamment le secret professionnel:

- de protéger, maintenir, restaurer et promouvoir la santé des personnes ou l'autonomie de leurs fonctions vitales physiques et psychiques, en tenant compte de la personnalité de chacune d'elles, dans ses composantes psychologique, sociale, économique et culturelle;

- de prévenir et évaluer la souffrance et la détresse des personnes et de participer à leur soulagement (que cette souffrance soit d'origine somatique, psychique ou sociale);

- de concourir au recueil des informations et aux méthodes qui seront utilisées par le médecin pour établir son diagnostic, par l'assistante sociale pour initier les démarches nécessaires, par les rééducateurs (ergothérapeutes, kinésithérapeutes, orthophonistes, etc.);

— de participer à l'évaluation du degré de dépendance des personnes ;

— de participer à la surveillance clinique des patients et à la mise en œuvre des thérapeutiques ; 
— de permettre à la personne malade d'intégrer l'expérience de la maladie dans son parcours de vie ;

- de favoriser le maintien, l'insertion ou la réinsertion des personnes dans leur cadre de vie familial et social;

- d'accompagner les patients en fin de vie et, en tant que de besoin, leur entourage.

De même, pour les médecins gériatre, les soins relationnels sont indispensables et possibles même avec des patients grabataires dits déments (Fossier-Varney, Morel-Vuilliez, \& Chapuy, 1999).

\section{Contexte de l'étude}

Notre hypothèse concerne l'importance de l'organisation du travail dans la sensation d'isolement des soignants, le manque de partage émotionnel et les risques pour la santé des soignants comme celle des résidents.

Afin de cerner le temps que peuvent passer les soignants auprès de leurs patients, mais également avec leurs collègues (de statuts similaires et autres), dans le but d'obtenir un débriefing émotionnel convenable et une bonne circulation des informations, nous allons présenter un panel de 40 observations ergonomiques de journées complètes, dans sept institutions gériatriques, Etablissement d'Hébergement pour Personnes Âgées Dépendantes (EHPAD) ou Unités de Soins de Longue Durée (USLD). Ces interventions ergonomiques ont été financées par les régions Ile-de-France et Rhône Alpes.

Différents types de structures prennent en charge les personnes âgées (Colvez, Ritchie, Ledésert, Ankri, Gardent, \& Fontaine, 1994). Ce secteur, à l'interface du médical et du social, a des contraintes spécifiques qui concernent notamment l'évaluation de l' «activité »: nombre de journées de prises en charge, degrés de dépendances et de besoins en soins en fonction de typologies de pathologies, évaluées à travers les groupes iso-ressources (GIR) et plus récemment l'information plus fine des coupes dites « Pathos » (Direction Générale de l'Action Sociale, 2006).

Dans notre panel, l'USLD de CHU héberge des résidents extrêmement dépendants (tous en GIR 1 ou 2) alors que les EHPAD comportent une part de résidents en GIR 3 ou 4, mais de moins en moins du fait du développement des alternatives à l'entrée en institution.

Des recommandations pour la qualité de l'accompagnement de la fin de vie sont médiatisées. En particulier la formation « Humanitude » (Gineste \& Pellissier, 2005), qui a fait l'objet d'émissions télévisées, est de plus en plus dispensée aux soignants des EHPAD. Cette formation propose des outils destinés à permettre « d'aider dans le respect et la tendresse ». Mais leurs possibilités de mise en œuvre semblent souvent limitées et ces formations conduisent à des injonctions paradoxales qui aggravent la souffrance des soignants, comme nous l'avons constaté dans les établissements de notre panel ayant participé auparavant à ces formations. Les recueils de données sur le travail réel ont pour objectif de montrer en quoi les personnels soignants travaillent ou non dans des conditions permettant cette volonté de prise en charge « humaine », évidemment partagée avant tout par le personnel soignant (Estryn-Béhar, 2008).

\section{2.- Méthode : Une démarche participative, visant à associer les soignants à l'amélioration de leurs propres conditions de travail}

Plus de la moitié de ces observations (23 sur 40) ont été réalisées par les soignants eux-mêmes, après avoir suivi une formation s'appuyant sur les observations des ergonomes. Nos observations concernent 14 infirmièr(e)s diplômés d'état (IDE) et 26 aides-soignant(e)s (AS) ou auxiliaires médico-psychologiques (AMP), regroupés sous la dénomination «AS » car faisant un travail identique. D’autres métiers ont été observés, qui ne sont pas détaillés ici. La grande majorité des soignants observés étant des femmes, le féminin sera utilisé.

La démarche suivie a été celle d'une ergonomie participative. Les ergonomes ont tout d'abord effec- 
tué leurs observations de journées complètes, les ont analysées, puis, après validation et enrichissement par les soignants observés, les ergonomes ont présenté leurs résultats devant un ensemble de soignants volontaires suivant une formation brève à l'analyse ergonomique, dans chaque établissement. La démarche ergonomique participative s'appuie sur le transfert de nombreuses connaissances ergonomiques dont l'assimilation est possible, dans une forme condensée, car elles sont illustrées par la réalité vécue par les soignants participant à la formation. La première étape consiste en deux jours de cours sur les connaissances ergonomiques de base, illustrées par la réalité locale observée. Elle a concerné les thématiques suivantes: charge physique, charge mentale ou cognitive et charge psychique ou émotionnelle, horaires et rythmes biologiques, environnement physique (bruit, température, éclairage), analyse de l'espace, liens organisation et qualité des soins. La deuxième étape est basée sur les observations, par les soignants eux-mêmes d'autres soignants de leur service, de métier et d'horaires différents, en utilisant les notions ergonomiques acquises. L'analyse conjointe des observations par les ergonomes et les soignants a facilité l'émergence des propositions.

La technique par observations ouvertes a été préférée à l'opposé des questionnaires trop directifs. L'échantillonnage de l'activité en action, événements ponctuels, a permis de rester factuel dans le relevé en éliminant le risque d'interprétation au cours du relevé. L'activité a été décrite en utilisant la classification admise dans ce secteur d'activité (SIIPS). Mais nous avons bien distingué les soins dits « directs », lorsqu'ils s'effectuent directement sur le patient ou sont préparés en sa présence, et les soins dits « indirects » qui sont représentés par toutes les préparations de soins hors présence du patient, comme la préparation de médicaments, de chariots de soins ou de changes et le rangement de matériel ainsi que sa décontamination.

Les soins à prodiguer sont surtout « techniques » pour les infirmières, et « de base » pour les aidessoignantes. D'autres activités viennent se greffer au long de la journée de chaque soignant. Ils ont à s'occuper de traçabilité des évolutions dans les dossiers de patients, de plannings de soins, de rendezvous à prendre auprès de médecins traitants, etc. Leur travail ne peut se dérouler sans partage des informations entre soignants de façon structurée ou sous forme d'interruption.

Les activités relevées ont été distinguées, par ailleurs, selon qu'elles sont le déroulement cohérent du travail en cours ou qu'elles représentent des interruptions de natures diverses (recherche de matériel, difficultés liées à l'organisation, recherche d'un membre du personnel, recherche d'informations, interruptions de la part des patients eux-mêmes).

Les interactions des différents éléments relevés et leurs déterminants ont été discutés spécifiquement en groupe de travail pour chaque établissement et unité d'hébergement étudiée. Nous avons cherché une compréhension des liens entre distances à parcourir, surfaces des locaux, équipements, effectifs, stabilité des équipes, horaires d'une part et disponibilité pour « allier compétence technique et humanité » d'autre part. Cette recommandation de bonne pratique est en effet l'introduction du guide préfacé en 2004 par le Directeur Général de la Santé et le Directeur Général de l'Action Sociale (Dab \& Trégoat, 2004), mais les obstacles à cette recommandation doivent être compris dans chaque situation locale.

Cependant, pour faire apparaitre l'importance des différences entre infirmières et aides soignantes, et pour permettre des préconisations plus générales, nous avons réalisé des moyennes pour chaque grade.

Deux objectifs étaient poursuivis. D'une part, ce croisement des regards permettait de minimiser l'impact de la connaissance de sa propre activité lors de l'observation d'un agent effectuant le même travail, évitant ainsi de pénaliser la qualité des relevés opérés. D'autre part, il avait pour objectif de permettre la compréhension du travail d'un autre professionnel pour que le groupe puisse dépasser, dans la recherche de propositions ultérieures, les a priori sur les difficultés, l'ampleur de l'implication de ces autres professionnels et éviter le repli catégoriel défensif. La démarche elle-même a contribué au renforcement des collectifs pluridisciplinaires. Durant ces observations, ont été relevés 
les divers lieux dans lesquels le soignant a besoin de se rendre pour travailler, les activités qu'il y pratique, s'il est interrompu, avec quels interlocuteurs il échange, ainsi que le contenu de certains échanges caractéristiques, les coopérations ou coactivités gênantes, avec le nombre de conversations simultanées, les postures qu'il est amené à adopter. Les interruptions ont été détaillées par grandes catégories, ont aussi été spécifiés la tâche initiale, la durée de l'interruption, le lieu de survenue et le devenir de la tâche initiale (reprise ou non). Dans certains cas les relevés ou leur dépouillement étaient incomplets expliquant les différences selon les tableaux présentés.

À la fin des observations par le personnel, les ergonomes ont aidé les soignants à dépouiller leurs observations de façon synthétique, aussi bien quantitativement que qualitativement, afin de les rendre intelligibles lors de réunions élargies au reste du service, à la direction et aux instances de dialogue social. À partir des données exhaustives, recueillies lors des observations de journées continues, il a été possible de comprendre les déterminants des arbitrages réalisés entre des contraintes, parfois difficilement conciliables, dans un contexte donné. Ces relevés systématiques sur l'ensemble d'un poste mettent en évidence les compromis élaborés par les personnels entre des prescriptions contradictoires et entre les critères d'efficacité et de qualité et la protection de leur santé. Les tableaux de déroulement de la journée de travail permettent d'analyser, ensemble, ce sur quoi il faudrait agir pour réduire les interruptions, les retards et les difficultés. Ils permettent aussi de mettre en valeur les savoir-faire et les compétences de chacun. Les éléments favorables à la qualité technique et relationnelle du travail et à la satisfaction professionnelle sont explicités. Quantitatives et qualitatives, ces données permettent de mettre en évidence la disponibilité réelle des soignants pour des prises en charge personnalisées, allant au rythme de la personne, et les opportunités formelles et informelles d'élaborations pluridisciplinaires pour réajuster les projets de soins.

Des tableaux de synthèse à trois colonnes (constats, risques ou avantages, propositions) ont été élaborés en groupe de travail. Ces données ont été synthétisées et approfondies par les ergonomes et les équipes afin d'élaborer collectivement des propositions architecturales, organisationnelles et techniques cohérentes entre elles, puis d'en étudier la faisabilité avec les directions. Lorsqu'un réaménagement architectural était envisagé, ou une nouvelle construction, un cahier des recommandations ergonomiques était préparé par notre équipe, en s'appuyant, en interne, sur les compétences d'une architecte spécialisée en architecture des lieux de travail. Ces propositions étaient débattues avec le groupe de travail puis transmises aux instances de l'établissement.

Nous ne détaillerons pas, ici, le travail des Agents de service (ASH) qui contribuent au ménage au suivi du linge et des repas, mais qui sont souvent sous la responsabilité d'un cadre non lié aux unités de soins, ce que nous avons remis en cause dans les préconisations de chaque établissement.

Nous présentons, ici, les résultats concernant la différence entre les affirmations de tous les projets d'établissement sur la « qualité des soins » garantie par une « prise en charge globale dans les meilleures conditions de sécurité et de confort » et la réalité de la parcellisation du travail et de l'isolement des soignants. Les recommandations co-élaborées, dans le cadre de cette recherche-action, pour chaque service, ne sont pas détaillées dans cette présentation.

\section{3.- Le lien entre échanges, éclatement des soins et isolement du soignant}

Parmi les données recueillies, la proportion de temps où le soignant est amené à travailler en silence est particulièrement frappante pour un métier qui attire pour son contenu relationnel. Huit IDE sur onze passent plus de $50 \%$ de leur temps sans échanger et 6 AS sur 14 sont dans cette situation. Une IDE peut atteindre 74,8\% de son temps de travail sans échanger et une AS 79,8\%. 


\section{1.- Le peu d'échanges entre professionnels montre l'isolement des soignants les uns par rapport aux autres, les temps de partage sont cependant nécessaires au bon déroulement des soins à prodiguer aux résidents}

En établissement de long séjour, les échanges de plus de cinq minutes sont exceptionnels au sein des équipes soignantes, tous métiers confondus, lors de partages collectifs. La majorité des concertations qui ont été observées a pour moyenne 1 minute 30 secondes d'échange continu, que ce soit entre personnel de statut identique ou de catégories différentes, dans les sept EHPAD ou USLD (Tableaux 1 et 2). Au sein des établissements gériatriques, les échanges pluridisciplinaires peuvent être restreints par la charge de travail et par le nombre de résidents à prendre en charge.

L'existence d'une seule IDE sur le même horaire est souvent la règle, de plus elle doit couvrir plusieurs ailes ou étages et réaliser, en série, un très grand nombre de soins prescrits. Par exemple dans un EHPAD public de province, les résidents hébergés ont un âge moyen de 85,9 ans. Ils sont $61 \%$ à être classés en groupe GIR 1 ou 2, les moins autonomes pour les actes de la vie. Leur état de santé est souvent sévère ou critique, puisqu'au cours des douze mois de l'année 2008, 102 sur 204 ont été hospitalisés. De plus, il y a eu au cours de la même année, 62 décès. Le besoin de soins infirmiers est illustré par le nombre de résidents pris en compte pour des traitements divers. Seuls 21 résidents sur 204 sont déclarés en absence de pathologie traitée. Par contre, des problèmes cardiovasculaires ont été traités dans l'année pour plus de 150 résidents. Des broncho-pleuro-pneumopathies ont été traitées chez 118 résidents. 30 patients sont diabétiques et 15 sont suivis pour dysthyroïdie. Les traitements pour troubles de la santé mentale sont extrêmement fréquents (état dépressif 83, états anxieux 97, psychose délire et hallucination 84, syndromes démentiels 11). Mais un seul IDE s'occupe de 94 ou 110 résidents, répartis en six unités, dans cet EHPAD.

La situation la moins défavorable que nous ayons observée a confié la responsabilité de seulement deux unités de 24 résidents à une même IDE, en USLD.

Dans ce contexte, les échanges collectifs, presque toujours sans médecin, se déroulent pendant moins de $10 \%$ du temps de travail pour sept infirmier(e)s sur 12 (moyenne 9,4\%) et pour neuf aidessoignant(e)s sur 17 (moyenne 13,8\%), les échanges collectifs des AS se déroulant le plus souvent entre AS seules, compte tenu de la difficulté organisationnelle de se coordonner de façon pluridisciplinaire mais également de l'absence de lieu satisfaisant.

Les transmissions entre équipes successives sont incluses dans ces échanges collectifs. Mais le plus souvent, l'infirmière partante synthétise des points ciblés sur quelques résidents pour l'infirmière arrivante, le temps restreint ne permet pas aux aides-soignants de s'exprimer. Ils assistent simplement et ne prennent la parole que pour un événement grave.

Les infirmières échangent autant avec des aides-soignantes qu'avec des infirmières, mais très peu avec les médecins, qui sont souvent des généralistes de ville. Chaque IDE travaille auprès des résidents qu'elle prend en charge, en association avec 4 à 18 AS, le jour. La nuit, les IDE sont responsables du double de patients, qui dorment partiellement mais ont des soins et sont changés, alors que les effectifs AS sont réduits au tiers de ce qu'il est de jour.

Les IDE parlent donc généralement plus avec des AS, que celles-ci avec une IDE, sauf lorsqu'il y a un IDE en sus de la seule IDE du matin et d'après-midi, qui travaille de 8 heures à 16 heures. Mais chaque AS a très peu d'échanges avec une IDE, puisque les échanges de l'IDE se répartissent sur 4 à 18 AS (deux AS par unité de 21 à 28 résidents, alors que l'IDE s'occupe quant à elle de 2 à 6 unités de jour et plus encore la nuit).

En moyenne les IDE ont parlé $\mathbf{7 , 8 \%}$ de leur temps de travail avec une AS en 22 échanges, soit une durée moyenne d'échange de 1 min 40 (mais 6 fois moins de 5\%). Ce sont principalement des échanges sur des faits le plus souvent non commentés, l'AS transmettant à l'IDE des points d'alerte médicaux «Mme X a des selles molles », « Mr Z a les talons rouges », « Mme W m'a griffée quand 
j'ai voulu la remonter sur son fauteuil », « je n'ai pas pu faire la douche à Mme A car j'ai dû rechanger Mme B, elle en avait mis partout! ». Les IDE ont parlé 5,1\% de leur temps avec une autre IDE en 11 échanges, d'une durée moyenne de 2 minutes.

Les aides-soignantes, qui travaillent en général à deux ou trois sur la même unité, échangent davantage entre elles (jusqu'à 20,8\% de leur temps de travail), qu'auprès d'infirmières (parfois seulement $1 \%$ de leur journée de travail) ou qu'avec des médecins (seules 3 AS sur 14 ont des échanges interindividuels avec un médecin, et seulement pour une durée de $0,3 \%$ à $2,4 \%$ de leur temps de travail). Les temps de concertations avec le personnel soignant en cours de poste sont limités (« tu m'aides à la lever? », «tu fais qui ? », « Te reste-t-il des lingettes? »). Elles prennent des nouvelles de l'avancement du travail, et de la manière dont celui-ci s'est déroulé (« elle n'était pas de bonne humeur ce matin », «ça va, il a été sage ?»), mais les difficultés sont insuffisamment évoquées. Dans le bureau infirmier, une AS et l'étudiante infirmière se montrent leurs blessures perpétrées par des résidents. En effet, lorsque des personnes démentes sont prises en charge trop rapidement, l'agitation des résidents s'aggrave. Les réactions de peur des personnes, ayant souvent des troubles du raisonnement (maladies d'Alzheimer et apparentées), peuvent entraîner des gestes ou paroles de défense (insultes, griffures, coups).

En moyenne les AS ont parlé 1,8\% de leur temps de travail avec une IDE en 5 échanges de durée moyenne $1 \mathrm{~min} 40$ (et seulement 2 fois plus de 5\% sur les 17 pour lesquelles l'information est disponible) et $0,3 \%$ avec un médecin. Elles ont parlé 9,6\% de leur temps avec une autre AS en 26 échanges. L'organisation de l'entraide pour le soulèvement des résidents à deux ou pour l'utilisation de la seule salle de douche est au cœur des échanges qui ne durent que 1 min30 en moyenne.

\section{2.- Ce sont les AS qui dialoguent le plus avec les résidents mais leurs constats sont peu utilisés pour reconsidérer régulièrement le projet de vie de chacun}

Les AS communiquent le plus avec les résidents ( 9 AS sur 17 parviennent à parler plus de $20 \%$ de leur temps de travail avec eux), mais elles n'échangent que peu, ou très peu, avec les IDE (de 0,0\% au minimum à $8,5 \%$ au maximum de leur temps de travail). Ceci peut s'expliquer par la nature des tâches à effectuer. Les AS ont en charge des soins de base (douches, toilettes, aide au repas) prenant bien plus de temps que la majorité des soins techniques réalisés par les IDE (distribution de comprimés, dosage de glycémie au bout du doigt, etc.). Les AS se retrouvent auprès des patients plus longtemps que les IDE, que ce soit au sein des chambres ou des lieux de vie. Les échanges ponctuent les gestes réalisés, préviennent et cherchent l'assentiment de la personne.

Mais, comme le suggère le nombre d'échanges avec les résidents, ces échanges sont majoritairement courts, décrivant simplement le soin (63 échanges pour 24,4\% du temps en moyenne, soit une durée moyenne d'échange de 1 min40). Tout est mis en œuvre par le personnel pour avoir des gestes doux et des paroles délicates, mais il est concrètement impossible d'attendre la participation ou même l'assentiment du résident. Le temps de séjour bref dans les chambres nécessite des gestes rapides et des échanges stéréotypés afin de demander les mouvements nécessaires, omettant les désirs spécifiques des résidents. Au-delà d'une apparente variété des temps d'échanges, en pratique, seuls les deux AS observées dans un des EHPAD publics, ont des temps d'animation réelle avec les résidents, avec des durées moyennes d'échanges dépassant 4 minutes. Elles échangent autour des travaux manuels effectués quotidiennement avec les résidents. Ces résidents sont incapables d'aller à une activité programmée dans un autre lieu, mais bénéficient fortement de cette animation au niveau de l'étage, cohérente avec leur état et leur besoin de stimulation. Le temps de déplacement est faible, du fait d'une architecture compacte et d'un nombre de résidents pris en charge inférieur d'un tiers à ce qui est observé dans les autres établissements.

Dans l'ensemble, on constate que lorsque le pourcentage du temps passé à parler avec les résidents 
dépasse $30 \%$, le nombre d'échanges est plus faible et la relation plus personnalisée. La situation la moins mauvaise est observée lorsque l'AS connaît les résidents, mais les changements d'affectation, souvent mensuels, réduisent les possibilités de soins personnalisés et de confiance. En revanche, dans une observation d'EHPAD à poste fixe pour au moins une année, une résidente qui se met à chanter pendant sa toilette est accompagnée par les AS. Les paroles peuvent porter sur le soin que l'AS va réaliser ( «allez, je vais vous laver »), chercher à faire participer la résidente physiquement (« donnez-moi votre main ») mais aussi psychiquement (« on remet cette robe aujourd'hui? »). Dans cet $\mathrm{EHPAD}$, où le nombre de résidents par AS est néanmoins élevé, l'AS prépare les petits-déjeuners sans avoir besoin de demander aux résidents, mais en aidant sa collègue novice ( « il faut lui mettre deux sucres »). En aidant une résidente à manger, l'AS veille à ce que tout se déroule correctement («c'est bon, ça vous plaît?»; «il n'est pas trop froid le café? »). On remarque ainsi une complicité entre le soigné et le soignant (« vous avez bien dormi? »- « oui ma caille »), même si les échanges restent généralement trop brefs pour chacun.

Ce sont rarement de vrais soins relationnels, d'échanges sans le support d'un soin, mais les AS, pendant ces différents temps de la journée, peuvent recueillir des informations importantes sur l'état physique du patient, mais aussi psychologique, permettant de constater des améliorations ou des dégradations de capacités motrices ou intellectuelles, accompagnées d'une possible amorce d'état dépressif associé ou non à un risque de dénutrition. Les informations recueillies ne sont pas toujours bien exploitées du fait d'un manque de partage des savoirs entre métiers, les AS ne sachant pas forcément interpréter un comportement anormal, par exemple dû à un surdosage médicamenteux. Nous constatons que, de façon générale, les AS ne trouvent que peu d'opportunités pour transmettre à l'IDE leurs notations; une recherche de l'IDE n'est entreprise que lors d'un événement grave (chute, diarrhée, etc.). Dans seulement 2 cas l'AS a pu échanger plus de 5\% du temps avec une IDE.

Pour les IDE, les échanges avec les résidents et familles ont été inférieurs à $20 \%$ du temps sauf dans un cas (IDE d'après-midi ayant « accompagné » le fils d'une résidente avant et après le décès). Le nombre d'épisodes (jusqu'à 103) montre leur brièveté, d'autant que les IDE prennent en charge davantage de résidents. De nombreux résidents ne sont pas vus pendant un poste par l'IDE, ou uniquement rapidement pendant la distribution des médicaments, quelques secondes ou dizaines de secondes par résident. En moyenne les IDE ont parlé $11,2 \%$ de leur temps de travail avec un résident, en 47 épisodes (durée moyenne 1 minute), et 1,9\% avec une famille, en 2 épisodes. Notons que le week-end, non observé, la situation aurait été un peu différente.

Soulignons que, pour ces IDE, les échanges avec les médecins sont aussi très limités (inexistants ou inférieurs à 3\% du temps de travail pour $10 \mathrm{IDE}$ sur 12). Le peu d'échanges AS/IDE entraîne clairement une perte d'informations au niveau des IDE qui sont pourtant les références pour les médecins et les familles, les AS ne parlant quasiment jamais à un médecin. Il existe alors un risque dans les soins à administrer aux résidents. L'ensemble des informations fragmentaires recueillies sur l'état de santé physique et psychologique des personnes, non partagées, ne permettent pas les ajustements nécessaires suffisamment précocement et peuvent être source d'erreurs potentiellement dangereuses pour la santé des résidents. Notons que les EHPAD du secteur privé non lucratif étudiés fonctionnent sans médecin présent sur site et font appel, en fonction des besoins, aux généralistes qui suivent individuellement les résidents.

Par ailleurs, le constat de dégradations progressives de l'état de personnes dépendantes, vivant dans ces EHPAD ou USLD leur fin de vie, est émotionnellement lourd pour les AS (exemple: «Laissezmoi mourir »). Elles en reparlent un peu, entre AS, au moment de pauses, de concertation en prise et fin de poste ou, rapidement, au détour d'un couloir (exemple: "Madame $Z$ a attendu son fils toute la journée et il n'est jamais venu »). De même l'IDE qui a accompagné un décès, et un fils bouleversé, n'en a quasiment pas reparlé en équipe, sauf de façon factuelle très brève. Le soignant isolé est encore plus à risque de «burnout» lorsqu'il ne peut plus partager avec ses collègues ce qu'il ressent au cours des tâches qu'il a à effectuer tout au long de son activité de travail, a fortiori lorsqu'il doit 
faire face à des décès ou à certains soins particulièrement lourds, en gériatrie.

\section{3.- L'éclatement des tâches soignantes en de multiples soins de base et très peu de soins relationnels}

L'analyse de la répartition des activités montre que le temps que passe un soignant en présence d'un résident est souvent limité à la réalisation rapide des soins de base et, à un moindre degré, techniques, sans qu'ils soient précédés ou suivi de temps relationnels et sans qu'il soit possible d'aller au rythme de la personne, tout en préservant au maximum son autonomie résiduelle.

Les séquences d'activité s'enchaînent, s'interrompent, s'inversent, sans que le soignant puisse parfois prendre une pause, sans pouvoir échanger auprès de ses collègues, ou alors uniquement au sein de couloirs, lors de recherches d'informations ou de matériel. Au total les infirmiers observés changent d'activité 204 fois au cours d'un poste et les aides soignants 206 fois, soit une fois toute les $2 \min 10$ pour les deux professions (Tableau 3). La parcellisation du travail, désorganisée par une coordination écourtée et des interruptions multipliées, réduit le temps continu auprès des patients et ne permet pas le développement des soins relationnels.

Ces soins relationnels ne sont que très rarement supérieurs à $5 \%$ du temps de travail, qu'ils soient prodigués par des AS ou des IDE (quatre cas de plus de 5\% pour les AS et trois pour les IDE observé(e)s). Ceci est regrettable, puisque c'est essentiellement durant ces moments d'échanges avec le résident que les soignants peuvent en apprendre le plus sur son état de santé psychologique, et que ces moments de partage et d'attention sont un des éléments les plus appréciés par tous les soignants, de tous les grades, dans la pratique de leur profession. Lorsque les AS se retrouvent seuls avec un résident, sans retard pour les autres soins, ils tentent de les entourer de façon optimale, s'ils ne changent pas d'étage chaque mois (la rotation des affectations est une pratique de gestion du personnel courante en EHPAD) et connaissent, ainsi, leurs habitudes, leurs goûts, leurs histoires familiales, et leurs problèmes de santé. Par exemple, l'une des AS parle avec une résidente plus valide de son maquillage puis, en lui enlevant sa couche, elle lui parle du retour d'un résident qu'elle apprécie: «c'est platonique, mais c'est joli ». Elle évoque la venue de sa fille aînée et l'encourage en lui disant que « ce nombre de visites n'est pas donné à tous ». Elle souligne sa bonne mémoire liée à sa profession de professeur d'espagnol. Ces échanges minimisent la place du change et le rendent moins humiliant et déprimant.

Les soins relationnels sont censés être le moment où le soignant aide le résident dans des soins éducatifs, dialogue avec lui, prend son temps pour le stimuler et l'écouter afin de dépister les douleurs évitables, les raisons d'une dénutrition, d'un trouble de l'équilibre et d'accompagner son évolution psychologique et de comprendre ses besoins. Or, ici, nous comprenons bien que les soins relationnels ne sont pas vraiment réalisables en lien avec le temps imparti aux soignants pour l'ensemble de leurs tâches. Le résident, une fois sa toilette achevée, est amené dans des salons pour prendre le petit-déjeuner et il est placé dans l'ordre d'arrivée sans considérer les affinités et les possibilités des patients de converser ensemble.

Les soins indirects, de préparation et rangement, durent environ la moitié du temps consacré aux soins directs pour les AS. Nous avons relevé de 7,3\% à 32,9\% du temps de travail consacré par un(e) AS aux soins indirects. Les soins directs (de base, techniques et relationnels) occupent eux, de 31,2\% à $63,1 \%$. Ils dépassent $40 \%$ du temps de travail pour 13 AS sur 14 , hormis celles de nuit. Seulement six AS dépassent 50\% de leur temps en soins de base directs, car elles ont aussi à réaliser des activités de ménage ou de vaisselle hors de la présence des résidents. Mais soins directs et indirects peuvent parfois être de durée très comparables, la part de préparation étant presque aussi importante que le fait de pratiquer le soin, telle une AS du matin en USLD de CHU avec 25,1\% pour les soins indirects et $32,5 \%$ pour les soins directs dont 32,6\% de soins de base. La nuit, le soignant peut même consacrer plus de temps au cours de son poste à la partie indirecte des soins, préparant pour ses collègues de jour, par rapport à la réalisation de soins directs $(17,2 \%$ contre $14,9 \%)$. 
En EHPAD, lorsqu'elles ont la responsabilité de 80 à 116 résidents, les IDE se concentrent sur les patients ayant besoin de soins plus lourds. Elles peuvent alors rester longuement avec ces patients. Mais cela se réalise au détriment des autres, qui sont sous la surveillance complète des AS. Pour les IDE, la durée de préparation et de rangement, hors présence du patient, est plus longue que leur réalisation (en regroupant soins de base et techniques) dans 5 cas sur 10. L'IDE gardant la charge de la préparation des piluliers et de la traçabilité, les AS distribuant les médicaments. Les IDE de nuit, aidant souvent les AS aux soins de base, sont ceux qui réalisent le plus de soins directs. Celles de jour consacrent de $12,6 \%$ à $33,5 \%$ de leur temps aux soins indirects et jamais plus de 27,8\% à des soins directs. Les IDE qui distribuent les médicaments peuvent alors avoir de nombreux échanges avec les résidents, mais sans établir de vraie relation, puisque les échanges sont au maximum d'une minute en moyenne. Les IDE en horaires coupés cumulent les difficultés de gestion de tous les soins techniques à prodiguer le matin et le soir, incluant une double distribution de médicaments en salle à manger. Leurs échanges avec les malades sont les plus brefs de toutes les observations réalisées en gériatrie. S'ajoute à ces tâches le temps de déplacement au sein des couloirs, les IDE ayant très souvent plusieurs unités, ailes, secteurs ou étages, à prendre en charge (ces instants de déplacement concernent ici de $8 \%$ à $19,1 \%$ du temps d'une journée de travail). La traçabilité sur un nombre de dossiers conséquent, même s'il ne s'agit pas d'écrire dans tous les dossiers des résidents sous sa responsabilité (44 à 210 résidents selon les situations et les horaires observés), occupe une part substantielle du temps de travail.

L'isolement du soignant en lien avec l'éclatement de ses activités, essentiellement entre soins indirects et soins directs, devient alors prégnant dans une journée de travail. La partie travail de bureau, qui se cumule aux soins indirects, comprend: téléphone, traçabilité et gestion des dossiers de soins de patients à mettre à jour. Nous avons pu constater que seul(e)s certain(e)s IDE peuvent s'accorder une pause lors de leur journée.

Le fait d'être constamment en mouvement renforce leur isolement avec l'impossibilité de partage et d'échange avec d'autres soignants, ne les rencontrant pratiquement que dans les couloirs, où ne s'échangent que de brèves paroles. Enfin le nombre de changement d'activité sur une journée ne permet pas de mémoriser les informations perçues, de les analyser et de proposer des actions adaptées. Le risque de commettre des erreurs dans l'administration des soins est important dans un tel contexte.

Ce phénomène, identique pour les infirmier(e)s et pour les aides-soignant(e)s, ne permet pas l'élaboration d'un travail collectif où le soignant serait soutenu par ses pairs et pourrait répondre sans doute ni inquiétude aux familles lui posant des questions sur l'état de santé d'un parent.

Les AS, quant à elles, sont très interrompues au cours d'une journée de travail, tout en en passant davantage de temps auprès des patients. Ce temps véritable auprès des patients à prodiguer des soins relationnels, de base ou techniques, peut s'élever à $63,1 \%$ pour l'une de nos AS observées. De nombreuses soignantes (AS ou IDE) viennent les interrompre durant leurs soins afin de recueillir, ou diffuser, des informations qu'elles n'ont pu se transmettre auparavant. Cette part importante de recherche de données est due, en partie, au peu de présence des AS aux transmissions de début de prise de poste, comme à celles de fin, ainsi qu'aux staffs ou réunions entre équipe. La présence des AS à ces transmissions est faible en lien avec une organisation du travail en inadéquation avec le temps que peuvent prendre les tâches de chaque soignant (par exemple, une AS du matin sera en charge de toilettes généralement de $6 \mathrm{~h} 40$ à 10 heures, et plus selon les jours et les nécessités, et ne pourra assister à une réunion prévue à $9 \mathrm{~h} 30$, les IDE ayant terminé leurs prises de sang, administrations de médicaments, etc.) et un espace non adapté pour accueillir le nombre de soignants présents au sein des locaux pour ces réunions d'équipe. Les AS se sentent alors parfois exclues, renforçant leur sentiment de «mise à l'écart », de non-reconnaissance et, par là même, d'isolement.

On retrouve en moyenne $49,4 \%$ du temps de travail consacrés aux soins directs par les AS contre $22,6 \%$ par les IDE dont les temps de préparation, de travail de bureau et de déplacement sont bien plus importants que le temps passé auprès des résidents. Ceci montre bien les risques liés à une 
concertation principalement entre aides soignantes avec un partage factuel, et seulement des événements graves, avec les IDE.

\section{4.- Les interruptions fréquentes empêchent une ébauche de relation}

Les interruptions ne proviennent que très rarement d'un résident ou d'une famille, en moyenne sur nos observations 5 interruptions pour les AS et 4 pour les IDE durant respectivement $2 \%$ et $3 \% \mathrm{du}$ temps de travail (Tableau 4). Les interruptions sont essentiellement liées à des recherches d'information ou des difficultés d'organisation, en moyenne sur nos observations 27 interruptions pour les AS et 45 pour les IDE durant respectivement $8 \%$ et $13 \%$ du temps de travail. La recherche de matériel manquant lors des soins de base ou techniques est aussi fréquente, on a relevé, en particulier, que l'interdiction, pour raisons d'hygiène, d'entrer le chariot de soins dans les chambres oblige un grand nombre d'entrées et sorties; mais ce nombre d'entrées et sorties devient incompatible avec le lavage des mains demandé à chaque épisode. Le très grand nombre d'interruptions est prégnant, il scinde l'activité, en empêche la continuité, notamment auprès du résident: souvent plus d'une cinquantaine d'interruptions, notamment du fait de recherches d'informations dues à un manque de concertation préalable. Les interruptions occupent, toutes catégories d'interruptions confondues $17 \%$ du temps de travail des IDE et $13 \%$ de celui des AS. Elles diminuent l'efficience du travail et sont aussi génératrices d'erreurs par omission ou oubli.

\section{5.- Présence dans les différents lieux : le fractionnement des temps de séjour}

Les IDE travaillent globalement moins de temps que les AS/AMP auprès des résidents, et de façon encore plus fractionnée et brève (Tableau 5). Aucune IDE n'est restée plus de 30\% de son temps de travail dans les chambres et salles de bain. En revanche, elles sont entrées en moyenne 187 fois dans un lieu différent, soit un changement de lieu de travail toutes les $2 \mathrm{~min} 30$. Elles passent en moyenne $21,9 \%$ de leur temps de travail dans les chambres mais en 51 épisodes de moins de 2 minutes. Elles donnent aussi des médicaments en salle à manger. Couvrant plusieurs unités et étages, elles passent dans les couloirs 15,6\% de leur temps. Elles sont censées suivre, pour chaque résident, les bonnes pratiques correspondant à un grand nombre de protocoles : prévention des chutes, des plaies et escarres, des infections, de la dépression, de la dénutrition, de la déshydratation... Elles notent les informations sur les dossiers individuels de chaque résident, mais le temps en poste de soins-bureau infirmier pour préparer, ranger, écrire et téléphoner occupe $41,5 \%$ en moyenne. Or, ces temps de travail au poste-bureau infirmier les éloignent des résidents d'une façon très décevante. Aussi les IDE n'apprécient souvent pas, de ce fait, de travailler en long séjour.

Les AS travaillent la majorité de leur temps auprès des résidents, dans les chambres, sanitaires, salons, salles à manger. Mais les déplacements et recherches de matériel ou d'informations dans les couloirs induisent un fractionnement des séjours dans les différents lieux (122 changements de lieux en moyenne, soit un changement de lieu toutes les $3 \min 40$ ). Plus de la moitié des AS entre plus de 50 fois dans une chambre, alors qu'elles sont responsables de 8 à 13 résidents, même pendant des demipostes. Le peu de temps dans le poste-bureau infirmier (5,6\% en 4 épisodes en moyenne) objective les faibles possibilités d'échanges longs entre AS et IDE. Les échanges brefs dans les couloirs, qui se révèlent être un lieu de travail important où elles passent 16,4\% de leur temps, ne sont évidemment pas propices à une élaboration collective: rapidité des échanges, manque de confidentialité...

\section{6.- Influence de la typologie du bâtiment sur la disponibilité des soignants pour l'aide aux résidents}

L'installation des ailes d'habitation sur plusieurs étages et l'organisation de bâtiments en ailes de grande longueur sont les éléments de structure générale qui, dans les analyses ergonomiques de tous les établissements étudiés, ont démontré le plus fort impact sur le travail des soignants. 
Dans tous les services présentant l'une ou l'ensemble de ces caractéristiques, les analyses ont montré comment l'augmentation des distances à parcourir et donc des temps de déplacement contraignent le personnel à une diminution du temps dédié aux soins directs et les résidents à une perte d'autonomie dans leurs déplacements. Ceci a, à son tour, un impact sur le travail du personnel.

Ces problèmes, liés à l'organisation sur plusieurs étages, peuvent en partie être soulagé par l'installation de moyens techniques adaptés. Mais les analyses montrent que l'utilisation des ascenseurs n'est compatible qu'avec l'installation d'un nombre conséquent d'ascenseurs, jamais retrouvé, et ces ascenseurs ne sont que peu utilisables, de façon autonome, par une population vieillissante.

Mais, la pénibilité due aux distances à parcourir, est plus dépendante des choix d'organisation de l'établissement (nombre de chambres dans chaque aile par rapport au nombre de personnel affecté, existence de douche dans le sanitaire de chaque chambre ou salle de bains commune parfois à plus de $60 \mathrm{~m}$ de la chambre la plus éloignée...) que de la typologie de bâtiment choisie. Une typologie monocouloir n'est pas nécessairement plus chronophage qu'une typologie à îlot central si son organisation interne permet le rapprochement des locaux de support de soins ainsi que des locaux de vie des chambres. L'îlot central peut s'avérer très chronophage si les équipes ne sont pas sectorisées et les locaux de support de soins y sont en général pénalisés par le manque de lumière.

\section{Exemple: Compréhension de l'impact des ascenseurs sur l'activité réelle des soignants}

Le manque d'ascenseur adapté au nombre de résidents en fauteuils roulants devant utiliser cet équipement pour se rendre au restaurant réduit le temps disponible pour les soins personnalisés: un seul ascenseur est prévu pour les 4 étages. Il est utilisé aussi pour les chariots du petit-déjeuner et les visiteurs. Deux fauteuils roulants et deux soignants peuvent prendre place en même temps dans l'ascenseur; de 14,6\% du temps de travail à 25,3\% du temps de travail des 6 demi-journées observées ( 3 postes en horaires coupés de 2 fois 5 heures avec 2 heures de coupure) se passent dans les couloirs halls et ascenseurs. On constate que le temps dans les couloirs, les halls, à attendre les ascenseurs, et dans ceux-ci, représente entre le tiers et les deux tiers du temps dans les chambres. Il faut attendre les arrêts d'ascenseurs qui s'ouvrent déjà occupés, à la descente comme à la montée.

Il faut essayer d'apaiser les résidents impatients en organisant les deux files de fauteuils roulants. Il y a jusqu'à 10 fauteuils rangés en file de deux fauteuils allant au même étage. La figure 1 permet de constater l'impact de l'ascenseur sur le temps disponible pour aider les résidents à s'alimenter, le transport et l'attente occupant, en effet, deux fois plus de temps que l'aide au repas.

\section{4.- Discussion}

\section{1.- L'isolement et la parcellisation du travail réduisent les possibilités de soins relationnels en gériatrie}

L'affirmation institutionnelle de la nécessaire « prise en charge globale » d'un patient et des «partenariats soignants / soignés » ne peut se concrétiser sans une élaboration collective d'un projet de soins et de vie. Le fait de ne pouvoir échanger réellement, ni avec le patient, ni avec les soignants, enferme ces derniers dans une organisation strictement individuelle, où chaque soignant effectue ses activités sans lien avec l'activité de l'autre et de façon morcelée. En effet, malgré la forte présence des AS auprès des patients, la qualité de l'échange peut ne pas être satisfaisante, compte tenu en particulier du nombre élevé d'interruptions, affaiblissant considérablement le temps passé de façon continue auprès d'un patient, ne permettant pas de l'amener à dialoguer davantage.

Parallèlement à la période où les observations ont été pratiquées, le « Manuel de Certification des Etablissements de Santé » insiste sur le fait que la prise en charge de certaines populations, dont les besoins sont spécifiques, représente un enjeu important en termes d'amélioration de la qualité des 
soins. Le premier groupe cité est celui des personnes âgées, suivi de celui des patients porteurs de maladies chroniques et des personnes atteintes d'un handicap ou qui sont démunies. Les EHPAD et Unités de soins de Longue Durée (USLD) n'hébergent que des personnes relevant de ces catégories. La priorité est donnée à la vérification qu' "Un projet de soins personnalisé est élaboré avec les professionnels concernés (projet de vie en USLD); Le projet de soins personnalisé est réajusté en fonction d'évaluations périodiques de l'état de santé du patient en impliquant le patient et s'il y a lieu l'entourage » (Direction de l'Amélioration de la Qualité et de la Sécurité des Soins, 2008).

Aussi, nous comprenons bien mieux les scores élevés de burnout qui sont relevés parmi les soignants de long séjour en France et pourquoi un soignant, quelle que soit sa profession, peut être insatisfait du service qu'il rend. Selon l'analyse statistique PRESST - NEXT, en France, les soignants de long séjour gériatrique sont $52,9 \%$ à avoir un score de burnout élevé contre $46 \%$ de l'ensemble des soignants. Le pourcentage de soignants avec un score élevé de burnout sur l'ensemble de l'échantillon européen est de $33 \%$ en long séjour gériatrique contre $27,8 \%$ de l'ensemble des spécialités. Le manque de satisfaction professionnelle des IDE en gériatrie est un problème aussi pour attirer et retenir un nombre suffisant d'IDE dans ces structures.

Pour compléter ce raisonnement, ajoutons que l'idée de maltraitance par le personnel soignant, notamment à l'égard des personnes âgées, sous-tend le développement d'injonctions sociétales pour permettre des prises en charge plus « humaines », dont le succès de la formation « humanitude » est le meilleur reflet. Mais la maltraitance est un «phénomène collectif et organisationnel, et non un comportement de malveillance individuel. Plus encore, si les patients sont maltraités, c'est avant tout parce qu'on maltraite le métier » (Litim \& Kostulski, 2006). Les soignants ont ce désir de prise en charge humaine, mais en ont-ils les possibilités?

\section{2.- L'importance du travail collectif}

L'importance de collectifs de travail soudés et faisant évoluer ensemble un projet de soins ou de vie personnalisé a été soulignée (Estryn-Behar, 2008). Mais, un collectif ne peut exister que s'il y a un espace et du temps pour une pratique réflexive. Il faut pouvoir mettre en controverse les différentes manières de pratiquer afin d'élaborer cette réflexion commune. Le temps passé par les soignants auprès de leurs patients, mais également avec leurs collègues (de statut similaire et autres), dans le but d'obtenir un débriefing émotionnel convenable et une bonne circulation des informations, améliore la qualité du travail fourni auprès des patients. L'importance des transmissions, staffs et réunions a été soulignée (Meißner, Hasselhorn, Estryn-Behar, Nezet, Pokorski, \& Gould, 2007). Considérer le malade dans sa globalité, éduquer le malade à l'hôpital pour une meilleure observance permettant la prévention des rechutes et des complications, nécessitent la réduction des incertitudes des soignants, aussi bien sur les aspects techniques que psychologiques des soins. La constitution de « collectifs de travail » soudés et la place des transmissions orales sont centrales pour la qualité des soins et la satisfaction professionnelle (Estryn-Behar, 2008). La continuité des soins dépend d'un transfert efficace d'informations entre équipes successives. De plus la transmission permet une réelle opportunité d'interaction sociale, de soutien émotionnel et de formation entre les soignants expérimentés et les novices (Kerr, 2002; Payne, Hardey, \& Coleman, 2000). Kaissi, Johnson et Kirschbaum (2003) ont souligné qu'une réduction substantielle des erreurs dans les soins ne saurait être obtenue tant que plus d'attention n'est pas donnée aux solutions tenant compte des facteurs humains, tels que l'amélioration du travail d'équipe et de la communication au sein des équipes soignantes.

Les discussions de groupe autour des observations dans les différents établissements, ont aussi montré la confrontation entre une volonté de prise en charge personnalisée et une prescription (protocoles, nombre de résidents) empêchant une élaboration pluridisciplinaire satisfaisante. Il apparait clairement qu'en EHPAD, seule une sectorisation avec un IDE référent par étage permet de se consacrer à 40 malades, d'avoir des dossiers complets à chaque étage et de suivre l'accompagnement en permettant un travail pluridisciplinaire associant les AS lors des transmissions et de la venue des 
médecins.

Les groupes de travail ont toujours conclu que les transmissions entre équipes successives associant IDE, AS et ASH, devaient être développées pour prendre suffisamment connaissance des états des patients et définir les priorités dès la prise de poste. Lorsque l'IDE reste seule pour plusieurs étages, il est nécessaire au moins de faire successivement une transmission à chaque étage associant les AS de l'étage. Pour cela il faut un lieu adéquat à chaque étage et augmenter le temps de chevauchement des IDE et AS. Si cette augmentation n'est pas possible pour les AS, il peut être envisagé de décaler au moins le chevauchement des AS, entre les étages, pour permettre aux IDE de les rencontrer successivement. Les agents proposent d'équilibrer entre les étages avec des résidents « très dépendants » et d'autres plus « valides ». Mais pour cela les aides mécaniques à la manutention doivent être disponibles (lèves-malades au sol ou au mieux lèves malades sur rail au plafond joignant la chambre et la salle de bains). Les soignants souhaitent aussi souder des équipes autour d'un groupe de résidents, avec groupe de parole, médecins référents et moins de médecins intervenants, staff mensuel sur les malades de chaque médecin généraliste extérieur intervenant pour réactualiser les prises en charges des résidents, notifier les difficultés rencontrées et rendre plus cohérentes les réponses. Le groupe de parole est souhaité par les soignants «pour valorisation des personnels confrontés à la souffrance et la mort ». Les agents proposent de travailler en collaboration avec des ASH stables sur les unités, et non de les laisser sous la responsabilité d'un encadrement commun pour toutes les unités indépendant du fonctionnement de ces unités. Cela apparaît nécessaire pour un équilibre dans la continuité des soins et pour optimiser la sécurité des résidents. En effet, les ASH sont encore souvent présentes sans formation alors que les personnes hébergées sont de plus en plus poly-pathologiques, il est proposé de prévoir un AS référant pour les ASH.

\section{3.- Eléments décisionnels et pistes de préconisations}

Les recherches-actions que nous avons conduites ont montré et rappelé l'intérêt d'une approche systémique qui prenne la mesure de l'injonction paradoxale concernant le travail collectif, le projet de vie personnalisé et régulièrement réévalué ou la création de nouveaux droits et principes pour permettre la plus grande visibilité et reconnaissance des personnes âgées.

Les premières pistes concernent les rapports entre le travail collectif, l'organisation du travail et les effectifs. Nous avons constaté qu'ils sont de plus en plus ambigus : travailler officiellement « en équipe » ne signifie pas systématiquement une réelle coopération et l'organisation du travail peut limiter volontairement mais aussi indirectement la coopération au travail (Gheorgiu \& Moatty, 2005). Cette ambiguïté s'actualise dans le secteur hospitalier notamment à travers l'injonction paradoxale de coopérer (Haute Autorité de Santé, 2008) tout en réduisant les possibilités : réduction des temps de chevauchement entre équipes, entrées en institution de résidents plus lourds avec un ratio personnel/ résidents identique, augmentation de la traçabilité, censée par ailleurs favoriser la coopération mais, de fait, très chronophage pour un bénéfice surtout médico-légal en cas de contestation. La facilitation de la coopération est fondamentale en long séjour. La coopération semble, dans différentes organisations, liée à la notion de dépendance mutuelle (De La Garza, 1995 ; Costes, Angros, \& Kerbal, 2006) et plus encore dans ces structures de soins.

Des échanges pluridisciplinaires nécessitent une liberté de parole d'autant plus dure à acquérir dans un milieu fortement hiérarchisé. De plus, la mutualisation des moyens humains est une solution courante pour des directions acceptant néanmoins l'idée d'un cercle vicieux: connaissance moindre des résidents et des services, pertes de temps, risques supplémentaires d'arrêts de travail et d'abandons...

Actuellement, les facteurs d'isolement s'accroissent avec la «protocolisation » qui s'accroît et la faiblesse des effectifs de long séjour rendant difficile la constitution de collectifs soignants soudés. Ces facteurs pourraient encore venir renforcer le phénomène d'isolement, formant un cercle vicieux par rapport à la parcellisation et au manque de temps à accorder aux patients que nous avons observés. 
Des préconisations peuvent être mises en place, même dans le contexte des effectifs actuel. Elles concernent la stabilité des affectations pour au moins un an, pour le non-recours aux horaires coupés et au temps partiel contraint et pour des temps de chevauchements suffisants pour un réel partage des informations et un débriefing émotionnel. En effet, la réduction attendue du temps perdu en interruptions pour rechercher les informations et le matériel peut compenser une partie du coût du chevauchement. De plus une évaluation avant/ après peut chercher à comparer le coût de ces organisations améliorées pour plus de travail d'équipe et le bénéfice obtenu par moins d'arrêts maladie une meilleure attractivité et fidélisation des soignants, permettant moins de recours à l'intérim et aux heures supplémentaires.

Cependant, des régulations sociétales, plus générales nécessaires, telles que le ratio de personnel en fonction du nombre de résidents ne dépendent pas des structures. En ce sens, une fois les spécificités locales évaluées, l'ergonomie devient une discipline politique, pour permettre une organisation moins pathogène. Cette question des effectifs paraît encore plus centrale dans les unités de longs séjours que dans d'autres unités de soins, alors que nous avons pu relever dans une structure qu'un quart d'effectif AS en plus augmentait de bien plus du quart le temps de présence auprès des résidents. Des situations meilleures existent et permettent de cerner les priorités à développer ailleurs. L'exemple de l'EHPAD public, où nous avons observé des temps d'animation réelle avec les résidents et des durées moyennes d'échanges avec eux dépassant 4 minutes a montré l'efficacité d'une organisation et d'un espace sectorisé pour une qualité de vies des résidents et des soignants. Le temps de déplacement faible, du fait d'une architecture compacte et d'un lieu de vie pour les repas et l'animation à l'étage pour 2 unités, associé à un nombre de résidents pris en charge inférieur d'un tiers à ce qui est observé dans les autres établissements sont des prérequis pour que la démarche d'amélioration de la qualité demandée aux EHPAD se développe. Dans l'ensemble, on constate que lorsque le pourcentage du temps passé à parler avec les résidents dépasse 30\%, le nombre d'échanges est plus faible et la relation plus personnalisée. La situation la moins mauvaise est observée lorsque l'AS connaît les résidents, mais les changements d'affectation, souvent mensuels, réduisent les possibilités de soins personnalisés et de confiance.

Le Plan de Solidarité Grand Age (PSGA) 2007-2012, présenté en 2006 par Philippe Bas, alors Ministre délégué à la Sécurité sociale, aux Personnes âgées, aux Personnes handicapées et à la Famille a déterminé une allocation des dotations budgétaires soins, en réservant le bénéfice du ratio personnel/résidents de 1/1 aux seuls établissements qui accueillent des résidents d'un niveau de dépendance très élevé, et a seulement fait progresser le ratio cible de 0,55 à 0,65 pour la majorité des établissements qui hébergent des personnes âgées. De fait, des ratios de 0,55/1 résident correspondent, après déduction des personnels administratifs et ouvriers, et en tenant compte des congés, à un IDE présent pour 58 résidents le matin et l'après-midi, et moitié moins la nuit, ainsi qu'à un AS présent pour 10 résidents le matin et l'après-midi, et deux tiers de moins la nuit. Ce calcul ne tient même pas compte des IDE en position de cadre et des ASH, affectés au ménage, au linge ou à la restauration qui sont, dans ce calcul, inclus parmi les AS. Pascal Champvert (2008), président de l'association des directeurs au service des personnes âgées, estime lui qu'il faut doubler le nombre de professionnels, pour atteindre le demi-million. Qui plus est, la Fédération hospitalière de France (FHF) a observé des ratios de 0,24 ; la FHH fixe un objectif à atteindre rapidement de 0,8 .

C'est aussi ce qu'exposait Trivalle (2003). Il insiste sur la nécessité d'équipes pluridisciplinaires en SSR SLD, et documente que la densité en personnel au lit du malade dans les services de gérontologie est insuffisante, même comparée aux normes en vigueur (0.6 agents par lit Moyen Séjour, et 0,4 SLD). Il montre que ces normes sont normes obsolètes, ne prenant pas en compte la poly dépendance plus fréquente observée dans les dernières années. De même, l'évaluation de Zarit, Stephens, Townsend, et Greene (1998, repris en France in Belimin et al., 2003) sur le fardeau des aidants familiaux de malades fait bien percevoir que lorsque ces malades arrivent en institution le fardeau déjà très élevé s'est encore accru. 
Il est grand temps de prendre en compte ce que des études internationales montraient depuis longtemps (Reuben, Borok, Wolde-Tsakik, Ershoff, Fishman, et al. 1995) et de mieux comprendre et évaluer les difficultés d'autonomie des populations accueillies dans les établissements (Gervais, 2010 ; Grille EVA, 2008) afin de définir des ratios personnels /résidents adéquats.

La deuxième piste concerne les liens entre contenu de l'activité, organisation et espace de travail qui doivent être étudiés simultanément.

Les amputations de l'activité (Clot, 2002) du fait de contraintes architecturales, techniques ou matérielles, entraînent un grand nombre de déplacements, de changements de lieux et des épisodes courts dans la prise en charge des résidents et les coordinations disciplinaires et pluridisciplinaires. Ces constats sont aisément partagés par les personnels observés, même si leur ampleur peut surprendre, sous réserve de prendre en compte les variabilités inhérentes aux jours de la semaine. Les directions sont également de plus en plus réceptives, notamment compte tenu de la pénurie croissante en personnel (abandons du métier, arrêts de travail).

Des préconisations revêtent une relative généralité: lèves malades permettant de lever le résident seul, se centrer sur la relation au résident sans demander l'aide du collègue, temps de chevauchements suffisants pour un débriefing émotionnel et des coordinations pluridisciplinaires, possibilité d'entrer des chariots dans les chambres suffisamment grandes pour ne pas toucher le lit, compte tenu des nombreuses entrées et sorties...

D'autres préconisations sont plus spécifiques. Par exemple, la possibilité de lieux adaptés aux échanges pluridisciplinaires est directement dépendante de possibilités architecturales parfois très limitées.

Le raccourcissement des distances est une priorité pour réduire les temps perdus en déplacement et approvisionnement.

L'augmentation du temps près des résidents qu'on peut en espérer est un gage de sécurité et participe à la prévention des événements indésirables (chutes, fuites...) en améliorant la rapidité d'intervention en cas d'urgence et la surveillance.

Les préconisations concernant la personnalisation du projet de vie régulièrement réévalué ou la création de nouveaux droits et principes pour permettre la plus grande visibilité et reconnaissance des personnes âgées (Berrut, 2009) ne peuvent se mettre en œuvre sans une réelle prise en compte des pistes précédentes. Soulignons que le secrétaire des affaires sociales de l'ONU, Mr Zelenev, ouvrant le $19^{\mathrm{e}}$ Congrès de IAGG, note la priorité à donner à l'établissement d'une déclaration des droits des personnes âgées: droits fondamentaux à assurer par l'ensemble des pays, recommandations centrées sur la maltraitance, différences liées au sexe, encourager l'augmentation des allocations de ressources pour personnes âgées, créer de nouveaux droits et principes, permettre la plus grande visibilité et reconnaissance des personnes âgées. Il a montré la double signification de la bientraitance en milieu médico social (prévention de la maltraitance et plus), et sur la formation et l'évaluation des pratiques professionnelles pour une politique de qualité (Bloch, 2009; Vetel et le bureau du Syndicat National de Gérontologie Clinique,2008). Mais l'évaluation ne peut refléter la réalité et permettre des améliorations que si elle ne porte pas sur des points isolés du contexte, elle doit réfléchir aux déterminants de la qualité de façon systémique.

\section{Apport de cette méthodologie ergonomique participative}

D'autres données mériteraient d'être présentées: nombre de postures pénibles (jusqu'à 250 pour les AS et IDE), temps debout et distances parcourues (jusqu'à 14 kilomètres pour certaines IDE, généralement entre 6 et 12 kilomètres quel que soit le métier), contenu détaillé des échanges interprofessionnels, travail des cadres (difficultés de gestions de plannings et de projets). Au-delà de la nécessité de démarches projets pour construire les compromis, ces éléments rappellent la difficulté, mais surtout l'utilité, d'une approche systémique adaptée.

La présentation de certains fondamentaux de l'ergonomie, théoriques et méthodologiques, puis les 
observations, leurs analyses et l'élaboration de propositions en groupe de travail associant soignants et ergonomes ont eu vocation à faire émerger des « acteurs ergonomiques » qui, dans une posture de référents rapidement initiés, portent le point de vue du travail réel dans le cadre des projets de l'établissement, qu'ils concernent le projet d'établissement et la modification de l'organisation du travail ou le réaménagement ou la construction de nouveaux EHPAD. C'est en ce sens que la conception est distribuée (Béguin, 2007) et plus uniquement soumise aux instances de dialogue.

\section{Perspectives}

Lors de nos interventions, la présentation par le groupe de travail soignants-experts ergonomes au CHSCT des établissements étudiés et la remise d'un rapport coécrit sur les préconisations concernant l'espace et le matériel dans la majorité des établissements étudiés ont permis des reconsidérations importantes au niveau local. Des investissements ont pu être programmés. Dans deux établissements, l'écriture d'éléments qualitatifs, à inclure au cahier des charges des constructions programmées, a été finalisée et les projets en tiennent principalement compte.

Les comparaisons, avant / après intervention, que nous espérons mettre en œuvre chercheront à montrer l'efficience de la prévention intégrée et de la démarche ergonomique participative pour le système sanitaire et social, pour les soignants et pour les bénéficiaires.

\section{REMERCIEMENTS}

Ce travail, faisant suite à l'étude statistique européenne Presst-Next, subventionnée par la Commission européenne, a bénéficié du soutien de l'Assistance publique - Hôpitaux de Paris, du Conseil régional d'Ile-de-France et du Conseil régional Rhône-Alpes. Les ergonomes ayant successivement participé à ce travail ont été rémunérés grâce à ces subventions.

\section{RÉFÉRENCES}

Bartels, S., Shea, D., Beck, C., Clark, T. Mansfield, J-C., Kapp, M., \& Ouslander, J. (2003). The American Geriatrics Society and American Association for Geriatric Psychiatry recommendations for policies in support of quality mental health care in U.S. nursing homes. Journal of the American Geriatrics Society, $51(9), 1299-1304$.

Bas, Ph. (2006). Plan solidarité grand âge 2007-2012: http://www.cnsa.fr/IMG/pdf/plan_solidarite_grand_ age.pdf

Béguin, P. (2007). Prendre en compte l'activité de travail pour concevoir. @ ctivité, 4(2), 107-114, http:// www.activites.org/v4n2/beguin-FR.pdf

Belmin, J., Chassagne, Ph., Gonthier, R., Jeandel, C., \& Pfitzenmeyer, P. (2003). Gérontologie pour le praticien. Paris: Masson

Berrut, G. (2009). Droit universel de la personne âgée: une feuille de route. La revue de gériatrie, 34, 807808.

Bloch, M-A. (2009). Evaluer pour accompagner, Editorial. La revue de Gériatrie, http://www. revuedegeriatrie.fr/2009/04/3789/

Carré, J-M. (2007). J'ai (très) mal au travail. Les films Grains de Sable, Canal +, Institut National de l'Audiovisuel.

Champvert, P. http://cubitus.senat.fr/bulletin/20080128/dependance.html

Clot, Y. (2002). La fonction psychologique du travail. Paris: PUF.

Colvez, A., Ritchie, K., Ledésert, B. Ankri, J., Gardent, H., \& Fontaine, A. (1994). «Cantou » et long séjour hospitalier : évaluation comparative de deux modes de prise en charge de la démence sénile. Paris: INSERM. 
Costes, C., Augros, B., \& Kerbal, A. (2006). Les facteurs de risques organisationnels dans un organisme social. Communisation présentée au $41^{\text {ème }}$ congrès de la SELF, Caen, France, Septembre.

Dab, W., Trégoat JJ, 2004. Accueil de la brochure Recommandations de bonnes pratiques de soins en EHPAD, 61 pages, page 2. http://www.revuedegeriatrie.fr/recos/bonn_pratic_ehpad.pdf

Davezies, P. (2007). Intensification. Danger: le travail rétréci. Santé \& Travail, 57, 30-33.

Daydé, M.-C. (2008). La relation d'aide en soins infirmiers, aspects réglementaires et conceptuels. Soins, 53, 731, 35-38.

Daydé, M.-C., Lacroix, M-L., Pascal, C., \& Salabaras Clergues, E. (2007). Relation d'aide en soins infirmiers. Savoir et pratique infirmière. Paris: Masson. SFAP

De La Garza, C. (1995). Gestions individuelles et collectives du danger et du risque dans la maintenance d'infrastructures ferroviaires. Thèse de doctorat en Ergonomie, Ecole Pratique des Hautes Etudes, Université Paris 5, Paris.

Dejours, C. (1998). Souffrance en France, la banalisation de l'injustice sociale. Paris: Seuil.

Direction de l'Amélioration de la Qualité et de la Sécurité des Soins. (2008). Manuel V2010 de certification des établissements de santé - publication: novembre 2008 - nouvelle maquette : décembre 2008. Paris: Haute autorité de santé.

Direction Générale de l'Action Sociale (2006). Circulaire DGAS/SD éC n²006-518 du 6 décembre 2006 relative aux formations à l'utilistion de l'outil Pathos dans les établissements d'hébergement pour personnes âgées dépendenes. http://www.sante.gouv.fr/fichiers/bo/2007/07/01 z0010048.htm.

Estryn-Béhar, M. (1996). Ergonomie hospitalière - théorie et pratiques. Paris: ESTEM- De Boeck édition.

Estryn-Béhar, M. (1997). Stress et souffrance des soignants à l’hôpital. Paris: ESTEM- De Boeck édition.

Estryn-Behar, M. (2001). Interface entre les normes et les référentiels, et l'activité de travail réelle des soignants. Actualités et dossiers en santé publique, la documentation Française, 35, 71-73.

Estryn-Béhar, M. (2008). Santé et Satisfaction des soignants au travail en France et en Europe. Rennes: Presses de l'Ecole des hautes Etudes en Santé Publique.

Estryn-Behar, M., Le Nézet, O., Loriol, M., Ben-Brik, E., \& Caillard, J.F. (2003). Le travail de soins dans les hôpitaux locaux -Miroir grossissant des difficultés actuelles. Gestions hospitalières, 429, 603-611.

Estryn-Behar, M., \& Poinsignon H. (1989). Travailler à l'hôpital. Paris: Berger Levrault ed.

Estryn-Béhar, M., Salbreux, R., Paoli, M.C., \& Le Nezet, O. (2007). La situation professionnelle des accompagnants dans les établissements des personnes âgées ou handicapées. La Revue de Gériatrie, 32(2), 95-110.

Estryn-Béhar, M., Van der Heijden, B.I.J.M., Oginska, H., Camerino, D., Le Nezet, O., Conway, P.M., Fry, C., \& Hasselhorn, H.M. (2007). The impact of social work environment, Teamwork Characteristics, Burnout, and personal Factors Upon Intent to Leave Among European Nurses. Medical Care, 45, 939950.

FHF http://www.amcehpad.fr/spip.php?article499

Fossier-Varney, N., Morel-Vuilliez, P., \& Chapuy, P.H. (1999). De la convivialité au soin relationnel avec des patients grabataires dits déments. La revue de Gériatrie, http:/www.revuedegeriatrie.fr/1999/10/2484/

Gervais, P. (2010). Méthodologie de l'étude d'évaluation de l'implantation du Système de mesure de l'autonomie fonctionnelle dans le secteur médico social français. La revue de gériatrie, 35, 235-244

Gineste, Y, \& Pellissier, J. (2005). Humanitude : Comprendre la vieillesse, prendre soin des Hommes vieux. PARIS: Armand Collin.

Gheorgiu, M.D. \& Moatty, F. (2005). Les conditions du travail en équipe. DRESS / CEE, série Études, 49. http://www.sante.gouv.fr/drees/s.erieetudes/pdf/serieetud49.pdf

Guide d'évaluation multi dimensionnelle GEVA (arrêté du 6 fév 2008) 
Haute Autorité de Santé, HAS (2008). Délégation, transferts, nouveaux métiers... Comment favoriser des formes nouvelles de coopération entre professionnels de santé? Recommandation HAS en collaboration avec l'ONDPS. http://www.anfiide.com/Documents/documents-utiles/cooperation_professionnels_ synthese.pdf

Huez, D. (2008). Souffrir au travail, comprendre pour agir. Edition: Privé.

Kaissi, A., Johnson, T., \& Kirschbaum, M.-S. (2003). Measuring Teamwork and Patient Safety Attitudes of High-Risk Areas. Nursing Economic, 21(5), 211-218.

Karasek, R.A., \& Theorell T. (1990). Healthy Work: Stress, Productivity and the Reconstruction of Working Life. New York: Basic Books.

Kerguelen, A. (2008). “Actogram Kronos” : un outil d'aide à l'analyse de l'activité. In H. Norimatsu, \& N. Pigem (Eds.), Les techniques d'observation en sciences humaines (pp. 142-158). Paris: Armand Colin.

Kerr, M., (2002). A qualitative study of shift handover practice and function from a socio-technical perspective. Journal of Advanced Nursing 37(2), 125-134.

Litim, M., \& Kostulski, K. (2006). Le diagnostic d'une activité complexe en gériatrie. Nouvelle Revue de Psychosociologie, 1, 45-54.

Meißner, A., Hasselhorn, H.-M., Estryn-Behar, M., Nezet, O., Pokorski, J., \& Gould, D. (2007). Nurses' perception of shift handovers in Europe -Results from the European Nurses' Early Exit Study. Journal of Advanced Nursing, 57(5), 535-542

Payne, S., Hardey, M., \& Coleman, P. (2000). Interactions between nurses during handover in elderly care. Journal of Advanced Nursing, 32(2), 277-285.

Reuben, D.B., Borok, G.M., Wolde-Tsadik, G., Ershoff, D.H., Fishman, L.K. et al. (1995). A randomized trial of comprehensive geriatric assessment in the care of hospitalized patients. The New England Journal of Medicine, 332, 1345-1350.

Rogez, I. (2009). Etablissements d'Hébergement pour Personnes Agées Dépendantes - Synthèse sur les conditions de travail et les actions de prévention. Les dossiers de l'ARACT Nord-Pas de Calais.

Siegrist, J. (1996). Adverse health effects of high effort - low reward conditions at work. Journal of Occupational Health Psychology, 1, 27-43.

Stephen, J., Bartels, M.D., Aricca, R., Dums, B-A., Thomas, E., Oxman, M-D., et al. (2004). Evidence-Based Practices in Geriatric Mental Health Care. Focus, 2, 268-281.

Trivalle, C. (2003). Hospitalisation des personnes agées, Gerontologie. Paris: Masson.

Vetel, JM. et le bureau du Syndicat National de Gérontologie Clinique (2008) La vérité si je mens. La revue de Gériatrie, Editorial. http://www.revuedegeriatrie.fr/2008/06/3683/

Villatte, R., Gadbois, C., Bourne, J.P., \& Visier, L. (1993). Pratiques de l'ergonomie à l'hôpital. Faire sien les outils du changement. Paris: Inter Editions Paris.

Zarit, S.H., Stephens, M.A.P., Townsend, A., \& Greene, R. (1998). Stress reduction for family caregivers: Effects of adult day care use. Journals of Gerontology: Social Sciences, 53, S267-S278.

Zelenev, S. (2009). La revue de gériatrie, 34, 809-810.

\section{RÉSUMÉ}

L'organisation du travail et l'espace déterminent fortement la manière dont les soignants peuvent réaliser leur tâche. Leur cœur de métier en gériatrie est de prodiguer des soins de base, techniques et relationnels à des résidents souvent très dépendants. Les expériences pilotes d'ergonomie participative présentées ont eu pour objectif la compréhension des déterminants des écarts entre travail prescrit et travail réel pour proposer des améliorations cohérentes pour tous.

Les observations de 40 journées complètes de travail d'aides soignants (AS ; $\mathrm{n}=26$ ) et d'infirmiers (IDE; $\mathrm{n}=14$ ) ont été menées par des ergonomes et 
des soignants en formation-action.

On constate que les soignants ne peuvent rester auprès des résidents que de courts épisodes (AS moyenne 44\% du temps en 68 séjours et IDE 22\% en 51 séjours). Le peu de temps passé dans le poste de soins pour les AS (5,9\% versus $41,5 \%$ pour les IDE) objective les faibles possibilités d'échanges AS /IDE (en moyenne les AS ont parlé 1,8\% de leur temps de travail avec une IDE), alors que ce sont les AS qui dialoguent le plus avec les résidents. Les constats des AS paraissent peu utilisés pour « reconsidérer régulièrement le projet de vie » de chaque résident.

Les soignants peuvent rarement partager leur charge émotionnelle avec leurs collègues. Le risque de se retrouver dans une situation d'isolement et d'épuisement professionnel est majeur.

Cette méthode permet de proposer, en groupe pluridisciplinaire, des améliorations des conditions de travail, de la coopération des personnels et de la prise en charge des résidents.

\section{MoTS CLÉS}

Ergonomie participative, isolement au travail, épuisement professionnel, soins gériatriques, qualité des soins

\section{RÉFÉRENCEMENT}

Estryn-Behar, M., Chaumon, E., Garcia, F., Milanini-Magny, G., Bitot, Th., Ravache, A.E., \& Deslandes, H. (2011). Isolement, parcellisation du travail et qualité des soins en gériatrie. Activités, 8(1), pp. 77-103, http://www.activites.org/v8n1/v8n1.pdf

Article soumis le 7 septembre 2010 accepté pour publication le 19 février 2011. 


\begin{tabular}{|c|c|c|c|c|c|c|c|c|c|c|c|c|c|c|}
\hline & & & & $\begin{array}{c}\text { EHPAD } \\
\text { Privé } \\
\text { non }\end{array}$ & $\begin{array}{c}\text { EHPAD } \\
\text { Privé } \\
\text { non }\end{array}$ & EHPAD & ublic 1 & EHPAD & oublic 2 & & EHPAD & ublic 3 & & $\begin{array}{c}\text { Moyenne } \\
\text { des }\end{array}$ \\
\hline Infirmier & & Matin & $\begin{array}{l}\text { Après- } \\
\text { midi }\end{array}$ & Jour & Jour & Nuit & Nuit & Matin & $\begin{array}{c}\text { Après- } \\
\text { midi }\end{array}$ & Matin & Matin & $\begin{array}{c}\text { Après- } \\
\text { midi }\end{array}$ & Nuit & IDE \\
\hline & $\%$ & ND & $16,0 \%$ & $11,8 \%$ & $10,8 \%$ & $9,0 \%$ & $6,5 \%$ & $13,0 \%$ & $2,1 \%$ & $17,4 \%$ & $7,8 \%$ & $5,2 \%$ & $12,7 \%$ & $11,2 \%$ \\
\hline & $\mathrm{Nb}$ & ND & 55 & 103 & 47 & 6 & 60 & 9 & 19 & 69 & 43 & 54 & 59 & 47 \\
\hline & $\%$ & ND & $0,0 \%$ & $0,0 \%$ & $2,4 \%$ & $0,0 \%$ & $0,0 \%$ & $0,6 \%$ & $2,7 \%$ & $0,1 \%$ & $0,0 \%$ & $15,4 \%$ & $0,0 \%$ & $1,9 \%$ \\
\hline & $\mathrm{Nb}$ & ND & 0 & 0 & 4 & 0 & 0 & 1 & 6 & 1 & 0 & 9 & 0 & 2 \\
\hline & $\%$ & $8,4 \%$ & $4,6 \%$ & $5,8 \%$ & $0,0 \%$ & $9,7 \%$ & $10,0 \%$ & $11,0 \%$ & $6,5 \%$ & $5,0 \%$ & $11,4 \%$ & $21,8 \%$ & $19,3 \%$ & $9,4 \%$ \\
\hline & $\mathrm{Nb}$ & 13 & 12 & 7 & 0 & 6 & 4 & 4 & 3 & 3 & 14 & 3 & 27 & 8 \\
\hline & $\%$ & $0,0 \%$ & $0,0 \%$ & $0,0 \%$ & $0,8 \%$ & $0,0 \%$ & $0,0 \%$ & $0,0 \%$ & $0,6 \%$ & $2,8 \%$ & $0,6 \%$ & $0,1 \%$ & $0,0 \%$ & $0,4 \%$ \\
\hline & $\mathrm{Nb}$ & 0 & 0 & 0 & 1 & 0 & 0 & 0 & 2 & 3 & 11 & 3 & 0 & 2 \\
\hline & $\%$ & $3,1 \%$ & $3,4 \%$ & $3,6 \%$ & $12,9 \%$ & $5,5 \%$ & $30,0 \%$ & $0,4 \%$ & $15,6 \%$ & $9,5 \%$ & $0,7 \%$ & $4,1 \%$ & $5,9 \%$ & $7,8 \%$ \\
\hline & $\mathrm{Nb}$ & 27 & 14 & 24 & 21 & 33 & 11 & 1 & 11 & 39 & 16 & 25 & 25 & 22 \\
\hline & $\%$ & $6,5 \%$ & $3,7 \%$ & $7,2 \%$ & $7,7 \%$ & $0,8 \%$ & $0,0 \%$ & $1,5 \%$ & $7,8 \%$ & $4,3 \%$ & $12,3 \%$ & $8,7 \%$ & $0,2 \%$ & $5,1 \%$ \\
\hline & $\mathrm{Nb}$ & 17 & 5 & 19 & 13 & 1 & & 3 & 6 & 12 & 23 & 29 & 1 & 11 \\
\hline & $\%$ & $2,1 \%$ & $1,0 \%$ & $6,2 \%$ & $0,0 \%$ & $0,0 \%$ & $0,0 \%$ & $1,1 \%$ & $1,7 \%$ & $7,7 \%$ & $1,3 \%$ & $2,2 \%$ & $0,0 \%$ & $2,0 \%$ \\
\hline & $\mathrm{Nb}$ & 4 & 4 & 6 & 0 & 0 & 0 & 2 & 2 & 9 & 6 & 6 & 0 & 3 \\
\hline & $\%$ & $0,0 \%$ & $2,0 \%$ & $8,3 \%$ & $1,4 \%$ & $0,2 \%$ & $3,0 \%$ & $6,5 \%$ & $4,0 \%$ & $5,1 \%$ & $2,5 \%$ & $0,9 \%$ & $6,7 \%$ & $3,4 \%$ \\
\hline & $\mathrm{Nb}$ & 0 & 8 & 4 & 3 & 1 & 3 & 7 & 15 & 17 & 8 & 7 & 27 & 8 \\
\hline & $\%$ & $0,8 \%$ & $2,7 \%$ & $2,7 \%$ & $0,2 \%$ & $0,0 \%$ & $0,0 \%$ & $9,3 \%$ & $6,3 \%$ & $0,0 \%$ & $4,0 \%$ & $1,8 \%$ & $0,2 \%$ & $2,3 \%$ \\
\hline & $\mathrm{Nb}$ & 6 & 7 & 10 & 1 & 0 & 0 & 6 & 7 & & 14 & 13 & 2 & 6 \\
\hline $\begin{array}{c}\text { Pas } \\
\text { d'échange }\end{array}$ & $\%$ & ND & $67,5 \%$ & $54,4 \%$ & $63,9 \%$ & $74,8 \%$ & $50,5 \%$ & $55,2 \%$ & $33,3 \%$ & $47,7 \%$ & $60,0 \%$ & $39,8 \%$ & $55,0 \%$ & $54,7 \%$ \\
\hline
\end{tabular}

Tableau 1. Echanges effectués entre les infirmiers de divers services de gérontologie et l'ensemble des personnes avec qui ils / elles ont pu s'entretenir lors d'une journée de travail (nombre d'échanges tenus et pourcentage du temps total de travail consacré)

Table 1. Verbal exchanges between registered nurses working in geriatric care (two long-stay departments in hospitals, five state nursing homes and two private nursing homes) and patients, families, other staff, nursing assistants and doctors. Phone, other, none. (Number of verbal exchange and percentage of total working time devoted to them.) 


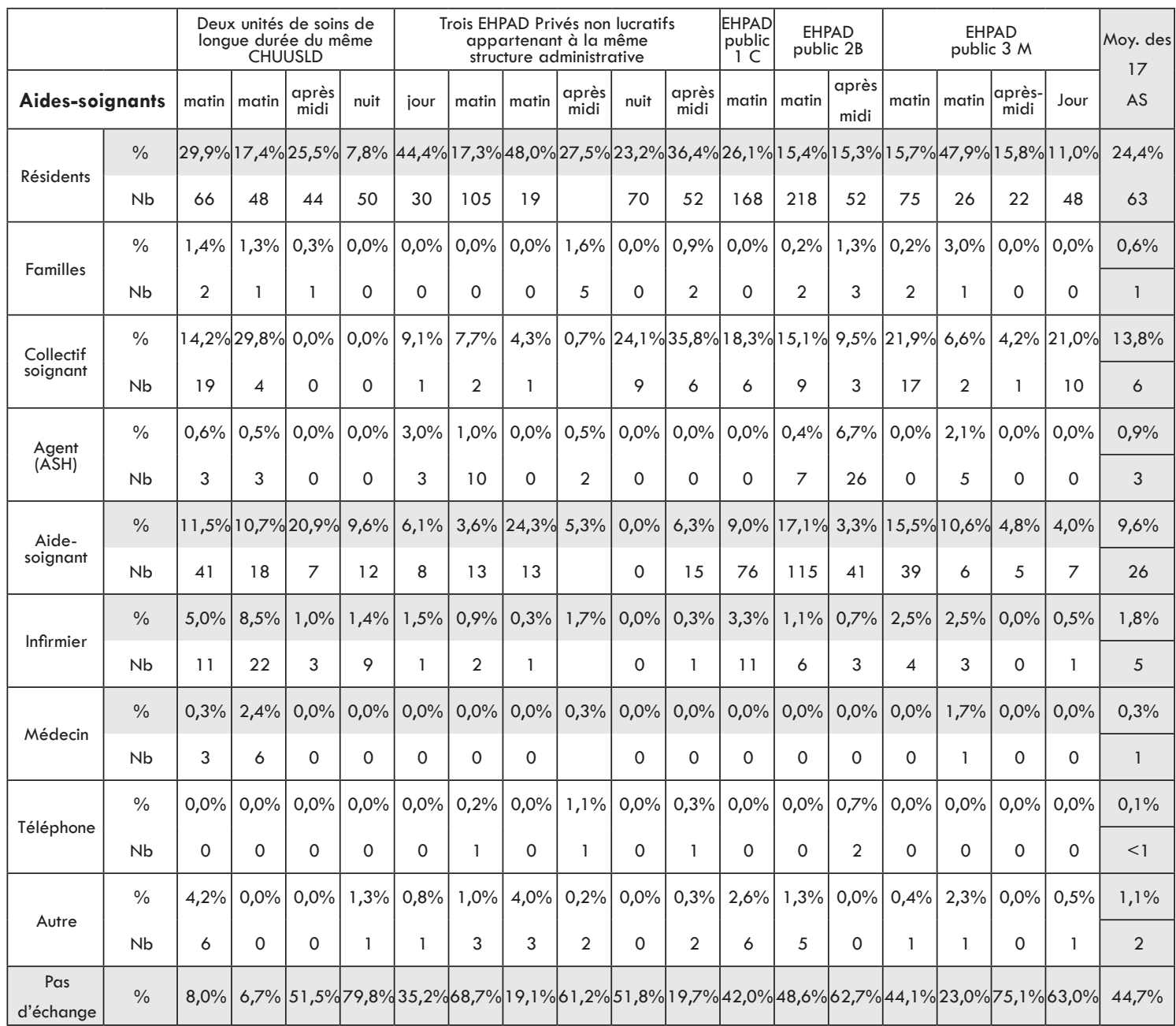

Tableau 2. Echanges effectués entre les aides soignants de divers services de gérontologie et l'ensemble des personnes avec qui ils / elles ont pu s'entretenir lors d'une journée de travail (nombre d'échanges tenus et pourcentage du temps total de travail consacré)

Table 2. Verbal exchanges between nursing assistants working in geriatric care (two long-stay departments in hospitals, five state nursing homes and two private nursing homes), and patients, families, other staff, nursing assistants and doctors. Phone, other, none. (Number of verbal exchange and percentage of total working time devoted to them.) 


\begin{tabular}{|c|c|c|c|c|c|c|c|c|c|c|c|c|c|c|c|c|}
\hline \multirow[b]{2}{*}{ Infirmiers } & \multirow[b]{2}{*}{ Horaires } & \multicolumn{2}{|c|}{$\begin{array}{l}\text { Soins relation- } \\
\text { nels }\end{array}$} & \multicolumn{2}{|c|}{$\begin{array}{l}\text { Soins tech- } \\
\text { niques }\end{array}$} & \multicolumn{2}{|c|}{ Soins de base } & \multicolumn{2}{|c|}{ Soins indirects } & \multicolumn{2}{|c|}{$\begin{array}{l}\text { Travail de } \\
\text { bureau }\end{array}$} & \multicolumn{2}{|c|}{ Concertation } & \multicolumn{2}{|c|}{$\begin{array}{l}\text { Déplacements } \\
\text { longs }\end{array}$} & \multirow{2}{*}{$\begin{array}{c}\mathrm{Nb} \\
\text { total } \\
\text { de } \\
\text { change- } \\
\text { ments } \\
\text { d'activité }\end{array}$} \\
\hline & & $\%$ & $\mathrm{Nb}$ & $\%$ & $\mathrm{Nb}$ & $\%$ & $\mathrm{Nb}$ & $\%$ & $\mathrm{Nb}$ & $\%$ & $\mathrm{Nb}$ & $\%$ & $\mathrm{Nb}$ & $\%$ & $\mathrm{Nb}$ & \\
\hline \multirow{2}{*}{ USLD } & M & $6,4 \%$ & 22 & $17,1 \%$ & 38 & $4,3 \%$ & 16 & $12,6 \%$ & 33 & $9,1 \%$ & 5 & $29,7 \%$ & 42 & $19,1 \%$ & 78 & 234 \\
\hline & $A M$ & $4,0 \%$ & 14 & $16,3 \%$ & 29 & $4,0 \%$ & 8 & $33,5 \%$ & 34 & $10,2 \%$ & 14 & $17,7 \%$ & 38 & $10,2 \%$ & 31 & 168 \\
\hline \multirow{3}{*}{$\begin{array}{l}\text { EHPAD public } \\
1 \mathrm{C}\end{array}$} & $A M$ & $11,5 \%$ & 39 & $11,4 \%$ & 37 & $4,2 \%$ & 19 & $12,0 \%$ & 56 & $28,3 \%$ & 62 & $29,8 \%$ & 45 & $0,0 \%$ & 0 & 258 \\
\hline & $\mathrm{N}$ & $4,8 \%$ & & $6,7 \%$ & & $13,3 \%$ & & $11,0 \%$ & & $20,7 \%$ & & $25,3 \%$ & & $14,2 \%$ & & ND \\
\hline & $\mathrm{N}$ & $0,3 \%$ & 1 & $7,7 \%$ & 19 & $19,0 \%$ & 51 & $15,2 \%$ & 24 & $23,7 \%$ & 5 & $30,9 \%$ & 9 & $5,0 \%$ & 39 & 148 \\
\hline \multirow{2}{*}{$\begin{array}{c}\text { EHPAD public } \\
2 \mathrm{~B}\end{array}$} & M & $1,7 \%$ & 1 & $16,2 \%$ & 11 & $1,3 \%$ & 2 & $19,7 \%$ & 15 & $16,8 \%$ & 12 & $13,9 \%$ & 9 & $14,1 \%$ & 33 & 83 \\
\hline & $A M$ & $6,1 \%$ & 3 & $13,2 \%$ & 33 & $6,5 \%$ & 6 & $23,0 \%$ & 17 & $23,2 \%$ & 18 & $16,5 \%$ & 10 & $15,4 \%$ & 28 & 115 \\
\hline \multirow{5}{*}{$\begin{array}{c}\text { EHPAD public } \\
3 \mathrm{M}\end{array}$} & M & $0,8 \%$ & 4 & $15,0 \%$ & 73 & $2,4 \%$ & 15 & $20,1 \%$ & 104 & $19,9 \%$ & 75 & $21,5 \%$ & 28 & $8,0 \%$ & 59 & 358 \\
\hline & M & $0,3 \%$ & 2 & $27,3 \%$ & 37 & $3,5 \%$ & 1 & $7,4 \%$ & 21 & $11,3 \%$ & 23 & $32,7 \%$ & 16 & $18,2 \%$ & 85 & 185 \\
\hline & $\mathrm{AM}$ & $17,4 \%$ & 20 & $2,5 \%$ & 36 & $0,5 \%$ & 4 & $12,5 \%$ & 21 & $15,2 \%$ & 35 & $28,6 \%$ & 9 & $23,1 \%$ & 49 & 174 \\
\hline & $\mathrm{N}$ & $0,3 \%$ & 3 & $3,8 \%$ & 13 & $0,0 \%$ & 0 & $12,0 \%$ & 9 & $2,4 \%$ & 11 & $52,2 \%$ & 11 & $29,2 \%$ & 56 & 103 \\
\hline & $\mathrm{N}$ & $1,0 \%$ & 6 & $9,3 \%$ & 38 & $9,3 \%$ & 26 & $10,6 \%$ & 31 & $14,0 \%$ & 44 & $25,2 \%$ & 45 & $16,6 \%$ & 90 & 280 \\
\hline $\begin{array}{l}\text { EHPAD Privé } \\
\text { non lucratif } \\
1 \mathrm{~V}\end{array}$ & J & $3,9 \%$ & 6 & $21,0 \%$ & 90 & $1,7 \%$ & 5 & $27,3 \%$ & 114 & $10,5 \%$ & 14 & $18,9 \%$ & 35 & $4,2 \%$ & 27 & 291 \\
\hline $\begin{array}{l}\text { E. Privé non } \\
\text { lucratif } 3 \mathrm{~S}\end{array}$ & J & $3,9 \%$ & 20 & $15,3 \%$ & 55 & $0,1 \%$ & 2 & $33,6 \%$ & 42 & $5,3 \%$ & 15 & $15,9 \%$ & 36 & $15,2 \%$ & 70 & 240 \\
\hline \multicolumn{2}{|c|}{ Moyenne des 14 IDE } & $4,5 \%$ & 11 & $13,1 \%$ & 39 & $5,0 \%$ & 12 & $17,8 \%$ & 40 & $15,0 \%$ & 26 & $25,6 \%$ & 26 & $13,8 \%$ & 50 & \\
\hline \multicolumn{2}{|l|}{ Moyenne } & \multicolumn{6}{|c|}{ Total Soins directs 22,6\% } & & & & & & & & & 204 \\
\hline
\end{tabular}

\begin{tabular}{|c|c|c|c|c|c|c|c|c|c|c|c|c|c|c|c|c|}
\hline \multirow[b]{2}{*}{$\begin{array}{l}\text { Aides soi- } \\
\text { gnants }\end{array}$} & \multirow[b]{2}{*}{ Horaires } & \multicolumn{2}{|c|}{$\begin{array}{c}\text { Soins } \\
\text { Relationnels }\end{array}$} & \multicolumn{2}{|c|}{$\begin{array}{l}\text { Soins tech- } \\
\text { niques }\end{array}$} & \multicolumn{2}{|c|}{ Soins de base } & \multicolumn{2}{|c|}{ Soins indirects } & \multicolumn{2}{|c|}{$\begin{array}{c}\text { Travail de } \\
\text { bureau }\end{array}$} & \multicolumn{2}{|c|}{ Concertation } & \multicolumn{2}{|c|}{$\begin{array}{c}\text { Déplacements } \\
\text { longs }\end{array}$} & \multirow{2}{*}{$\begin{array}{l}\mathrm{Nb} \text { total } \\
\text { de chan- } \\
\text { gements } \\
\text { d'activité }\end{array}$} \\
\hline & & $\%$ & $\mathrm{Nb}$ & $\%$ & $\mathrm{Nb}$ & $\%$ & $\mathrm{Nb}$ & $\%$ & $\mathrm{Nb}$ & $\%$ & $\mathrm{Nb}$ & $\%$ & $\mathrm{Nb}$ & $\%$ & $\mathrm{Nb}$ & \\
\hline \multirow{2}{*}{ USLD } & $M$ & $1,8 \%$ & 11 & $0,1 \%$ & 1 & $32,6 \%$ & 62 & $25,1 \%$ & 72 & $2,6 \%$ & 2 & $26,5 \%$ & 48 & $4,8 \%$ & 42 & 238 \\
\hline & $M$ & $2,0 \%$ & 16 & $0,2 \%$ & 1 & $41,2 \%$ & 47 & $17,7 \%$ & 45 & $5,8 \%$ & 3 & $24,7 \%$ & 11 & $4,5 \%$ & 25 & 148 \\
\hline \multirow{2}{*}{ USLD } & $\mathrm{AM}$ & $0,0 \%$ & 0 & $0,0 \%$ & 0 & $47,3 \%$ & 42 & $18,1 \%$ & 49 & $0,0 \%$ & 0 & $24,9 \%$ & 8 & $4,9 \%$ & 10 & 109 \\
\hline & $N$ & $2,2 \%$ & 5 & $0,0 \%$ & 0 & $29,0 \%$ & 48 & $14,0 \%$ & 31 & $3,0 \%$ & 3 & $9,5 \%$ & 9 & $1,8 \%$ & 9 & 105 \\
\hline \multirow{3}{*}{$\begin{array}{l}\text { EHPAD public } \\
\text { IC }\end{array}$} & $M$ & $4,1 \%$ & 40 & $0,0 \%$ & 0 & $52,4 \%$ & 95 & $11,2 \%$ & 66 & $1,2 \%$ & 7 & $22,0 \%$ & 48 & $6,5 \%$ & 45 & 301 \\
\hline & $M$ & $0,0 \%$ & 0 & $0,0 \%$ & 0 & $60,2 \%$ & 37 & $9,4 \%$ & 21 & $9,1 \%$ & 2 & $19,7 \%$ & 17 & $1,7 \%$ & 6 & 83 \\
\hline & A Cantou & $0,0 \%$ & 0 & $0,0 \%$ & 0 & $45,7 \%$ & 42 & $28,9 \%$ & 38 & $10,0 \%$ & 1 & $12,0 \%$ & 6 & $0,0 \%$ & 0 & 87 \\
\hline \multirow{2}{*}{$\begin{array}{l}\text { EHPAD public } \\
2 \text { B }\end{array}$} & $M$ & $2,8 \%$ & 39 & $0,6 \%$ & 3 & $50,6 \%$ & 194 & $8,4 \%$ & 73 & $4,0 \%$ & 5 & $13,4 \%$ & 53 & $4,9 \%$ & 71 & 438 \\
\hline & A & $2,8 \%$ & 12 & $2,0 \%$ & 12 & $58,3 \%$ & 100 & $7,3 \%$ & 17 & $0,0 \%$ & 0 & $5,3 \%$ & 8 & $5,1 \%$ & 31 & 180 \\
\hline \multirow{4}{*}{$\begin{array}{l}\text { EHPAD public } \\
3 \mathrm{M}\end{array}$} & M & $5,6 \%$ & 37 & $0,1 \%$ & 1 & $36,8 \%$ & 111 & $11,6 \%$ & 33 & $0,0 \%$ & 0 & $28,0 \%$ & 53 & $7,5 \%$ & 55 & 290 \\
\hline & $M$ cantou & $34,1 \%$ & 33 & $0,0 \%$ & 0 & $12,9 \%$ & 12 & $28,6 \%$ & 17 & $5,5 \%$ & 4 & $9,0 \%$ & 6 & $15,0 \%$ & 6 & 78 \\
\hline & $\mathrm{AM}$ & $17,4 \%$ & 22 & $0,0 \%$ & 0 & $10,0 \%$ & 9 & $32,9 \%$ & 32 & $2,0 \%$ & 3 & $13,8 \%$ & 13 & $28,7 \%$ & 30 & 109 \\
\hline & $M$ & $5,0 \%$ & 16 & $1,0 \%$ & 2 & $42,0 \%$ & 72 & $9,0 \%$ & 22 & $1,0 \%$ & 2 & $30,0 \%$ & 10 & $16,0 \%$ & 55 & 179 \\
\hline \multirow{6}{*}{$\begin{array}{c}\text { EHPAD Privé } \\
\text { non lucratiff } \\
1 \mathrm{SG}\end{array}$} & $M(5 \mathrm{~h} / 10)$ & $1,8 \%$ & 10 & $0,1 \%$ & 1 & $51,2 \%$ & 105 & $23,8 \%$ & 105 & $0,4 \%$ & 2 & $7,6 \%$ & 4 & $12,1 \%$ & 26 & 253 \\
\hline & $M(5 \mathrm{~h} / 10)$ & $2,3 \%$ & 10 & $0,1 \%$ & 1 & $60,4 \%$ & 50 & $15,9 \%$ & 38 & $1,1 \%$ & 3 & $13,0 \%$ & 11 & $2,5 \%$ & 5 & 118 \\
\hline & $M(5 \mathrm{~h} / 10)$ & $4,4 \%$ & 24 & $0,0 \%$ & 0 & $48,0 \%$ & 79 & $20,1 \%$ & 58 & $1,9 \%$ & 3 & $8,7 \%$ & 6 & $13,1 \%$ & 26 & 196 \\
\hline & $\mathrm{AM}(5 \mathrm{~h} / 10)$ & $22,2 \%$ & 23 & $0,0 \%$ & 0 & $55,9 \%$ & 147 & $10,0 \%$ & 38 & $1,4 \%$ & 4 & $10,5 \%$ & 21 & $0,0 \%$ & 0 & 233 \\
\hline & $\operatorname{AM}(5 \mathrm{~h} / 10)$ & $15,0 \%$ & 25 & $0,0 \%$ & 0 & $32,1 \%$ & 243 & $15,8 \%$ & 131 & $1,5 \%$ & 2 & $10,8 \%$ & 14 & $24,8 \%$ & 63 & 478 \\
\hline & $\operatorname{AM}(5 \mathrm{~h} / 10)$ & $11,5 \%$ & 20 & $2,4 \%$ & 12 & $39,4 \%$ & 104 & $4,0 \%$ & 18 & $1,0 \%$ & 1 & $42,0 \%$ & 20 & $3,0 \%$ & 9 & 184 \\
\hline \multirow{4}{*}{$\begin{array}{c}\text { EHPAD Privé } \\
\text { non lucratif } \\
2 \mathrm{~V}\end{array}$} & $\begin{array}{l}\text { J can- } \\
\text { toul } 1 \mathrm{~h} 40\end{array}$ & $23,7 \%$ & 5 & $1,9 \%$ & 3 & $25,7 \%$ & 38 & $10,1 \%$ & 17 & $2,1 \%$ & 2 & $7,2 \%$ & 4 & $11,2 \%$ & 26 & 95 \\
\hline & $\mathrm{J} 12 \mathrm{~h} 30$ & $4,4 \%$ & 39 & $3,0 \%$ & & $52,6 \%$ & 199 & $13,1 \%$ & 46 & $2,2 \%$ & 13 & $14,0 \%$ & 48 & $1,8 \%$ & 14 & 359 \\
\hline & $\mathrm{N} 10 \mathrm{~h} 40$ & $2,9 \%$ & 33 & $2,0 \%$ & 20 & $22,9 \%$ & 276 & $19,9 \%$ & 113 & $0,0 \%$ & 0 & $22,9 \%$ & 7 & $8,1 \%$ & 54 & 503 \\
\hline & $\mathrm{J} 12 \mathrm{~h}$ & $4,7 \%$ & 6 & $0,0 \%$ & 0 & $40,8 \%$ & 79 & $10,0 \%$ & 22 & $0,0 \%$ & 0 & $25,1 \%$ & 13 & $14,1 \%$ & 14 & 134 \\
\hline EHPAD & $\operatorname{AM}(6 / 10)$ & $6,2 \%$ & & $0,0 \%$ & & $33,6 \%$ & & $1,1 \%$ & & $13,1 \%$ & & $26,0 \%$ & & $20,0 \%$ & & ND \\
\hline Privé NL 35 & $\mathrm{~J} 10 \mathrm{~h}$ & $5,2 \%$ & 5 & $0,0 \%$ & 0 & $60,1 \%$ & 37 & $13,5 \%$ & 5 & $8,1 \%$ & 2 & $10,0 \%$ & 1 & $3,1 \%$ & 4 & 54 \\
\hline \multicolumn{2}{|c|}{ Moyenne des 25 AS } & $7,2 \%$ & 18 & $0,5 \%$ & 2 & $41,7 \%$ & 93 & $15,2 \%$ & 42 & $3,1 \%$ & 3 & $17,4 \%$ & 18 & $8,6 \%$ & 26 & \\
\hline \multicolumn{2}{|c|}{ Moyenne } & \multicolumn{5}{|c|}{ Total Soins directs $49,4 \%$} & & & & & & & & & & 206 \\
\hline
\end{tabular}

Tableau 3. Temps passé aux divers types de soins et d'activités autres dans une journée de travail par les infirmiers et aides soignants de divers services de gérontologie (nombre de soins réalisés et pourcentage du temps total de travail consacré)

Table 3. Time devoted to various kinds of care and other activities by registered nurses and nursing assistants working in geriatric care (two long-stay departments in hospitals, five state nursing homes and two private nursing homes). (Number of periods for each activity and percentage of total working time devoted to them.) 


\begin{tabular}{|c|c|c|c|c|c|c|c|c|}
\hline & \multicolumn{2}{|c|}{$\begin{array}{l}\text { Interruptions } \\
\text { par résidents ou } \\
\text { familles }\end{array}$} & \multicolumn{2}{|c|}{$\begin{array}{l}\text { Interruptions pour } \\
\text { problèmes de } \\
\text { matériel }\end{array}$} & \multicolumn{2}{|c|}{$\begin{array}{c}\text { Interruptions } \\
\text { pour recherches } \\
\text { d'information ou } \\
\text { difficultés d'orga- } \\
\text { nisation }\end{array}$} & \multicolumn{2}{|c|}{$\begin{array}{l}\text { Total des interrup- } \\
\text { tions sur le poste }\end{array}$} \\
\hline & $\mathrm{Nb}$ & $\begin{array}{c}\% \\
\text { du temps }\end{array}$ & $\mathrm{Nb}$ & $\begin{array}{c}\% \\
\text { du temps }\end{array}$ & $\mathrm{Nb}$ & $\begin{array}{c}\% \\
\text { du temps }\end{array}$ & $\mathrm{Nb}$ & $\begin{array}{c}\% \\
\text { du temps }\end{array}$ \\
\hline Infirmiers & 0 & & 0 & & & & & \\
\hline USLD matin & 0 & & 0 & & 56 & $15 \%$ & 56 & $15 \%$ \\
\hline USLD matin & 0 & & 0 & & 50 & $24 \%$ & 50 & $24 \%$ \\
\hline USLD matin & 0 & & 0 & & 58 & $14 \%$ & 58 & $14 \%$ \\
\hline USLD ap-midi & 0 & & 0 & & 65 & $11 \%$ & 65 & $11 \%$ \\
\hline $\begin{array}{r}\text { EHPAD Privé non lucratif } \\
\text { jour }\end{array}$ & 9 & $4 \%$ & 3 & $1 \%$ & 24 & $13 \%$ & 36 & $18 \%$ \\
\hline $\begin{array}{r}\text { EHPAD Privé non lucratif } \\
\text { jour }\end{array}$ & 1 & $1 \%$ & 2 & $1 \%$ & 4 & $4 \%$ & 7 & $6 \%$ \\
\hline EHPAD public matin & 8 & $6 \%$ & 5 & $2 \%$ & 11 & $9 \%$ & 24 & $17 \%$ \\
\hline EHPAD public après-midi & 14 & $4 \%$ & 12 & $7 \%$ & 45 & $20 \%$ & 71 & $31 \%$ \\
\hline EHPAD public matin & 3 & $12 \%$ & 3 & $1 \%$ & 18 & $11 \%$ & 24 & $24 \%$ \\
\hline EHPAD public après-midi & 5 & $3 \%$ & 2 & $3 \%$ & 20 & $17 \%$ & 27 & $23 \%$ \\
\hline EHPAD public après-midi & 4 & $1 \%$ & 12 & $1 \%$ & 60 & $6 \%$ & 76 & $8 \%$ \\
\hline Moyenne des 11 IDE & 4 & $3 \%$ & 4 & $1 \%$ & 37 & $13 \%$ & 45 & $17 \%$ \\
\hline
\end{tabular}

\begin{tabular}{|c|c|c|c|c|c|c|c|c|}
\hline Aides soignants & & & & & & & & \\
\hline USLD matin & 0 & & 0 & & 11 & $5 \%$ & 11 & $5 \%$ \\
\hline USLD après-midi & 0 & & 0 & & 7 & $22 \%$ & 7 & $22 \%$ \\
\hline USLD matin & 0 & & 0 & & 2 & $10 \%$ & 2 & $10 \%$ \\
\hline $\begin{array}{r}\text { EHPAD Privé non lucratif } \\
\text { après-midi }\end{array}$ & 14 & $11 \%$ & 5 & $3 \%$ & 5 & $3 \%$ & 24 & $17 \%$ \\
\hline $\begin{array}{r}\text { EHPAD Privé non lucratif } \\
\text { jour }\end{array}$ & 9 & $4 \%$ & 2 & $3 \%$ & 24 & $13 \%$ & 35 & $20 \%$ \\
\hline EHPAD public matin & 9 & $2 \%$ & 4 & $1 \%$ & 18 & $5 \%$ & 31 & $8 \%$ \\
\hline EHPAD public matin & & & 8 & $3 \%$ & 2 & $3 \%$ & 11 & $6 \%$ \\
\hline EHPAD public après-midi & 9 & $4 \%$ & 4 & $3 \%$ & 2 & $8 \%$ & 15 & $15 \%$ \\
\hline EHPAD public matin & 4 & $1 \%$ & 10 & $8 \%$ & 18 & $2 \%$ & 32 & $11 \%$ \\
\hline EHPAD public matin & 16 & $4 \%$ & 31 & $6 \%$ & 45 & $17 \%$ & 92 & $27 \%$ \\
\hline EHPAD public matin & 2 & $1 \%$ & 25 & $4 \%$ & 7 & $2 \%$ & 34 & $7 \%$ \\
\hline Moyenne des 11 AS & 5 & $2 \%$ & 8 & $3 \%$ & 13 & $8 \%$ & 27 & $13 \%$ \\
\hline
\end{tabular}

Tableau 4. Répartition des interruptions relevées au cours d'observations de journées complètes de travail d'aides soignants et d'infirmiers en gériatrie

Table 4. Interruptions, classified by category, observed during real-time task analysis of full days worked by registered nurses and nursing assistants involved in geriatric care. 


\begin{tabular}{|c|c|c|c|c|c|c|c|c|c|c|c|c|}
\hline \multirow[b]{2}{*}{ Infirmiers } & \multirow[t]{2}{*}{ horaire } & \multicolumn{2}{|c|}{$\begin{array}{c}\text { Chambres et leurs } \\
\text { sanitaire }\end{array}$} & \multicolumn{2}{|c|}{$\begin{array}{c}\text { Salon - salle à } \\
\text { manger }\end{array}$} & \multicolumn{2}{|c|}{ Couloirs escaliers } & \multicolumn{2}{|c|}{ Office } & \multicolumn{2}{|c|}{ Poste infirmier } & \multirow{2}{*}{$\begin{array}{l}\text { Nombre total } \\
\text { de change- } \\
\text { ments de lieux }\end{array}$} \\
\hline & & $\%$ & $\mathrm{Nb}$ & $\%$ & $\mathrm{Nb}$ & $\%$ & $\mathrm{Nb}$ & $\%$ & $\mathrm{Nb}$ & $\%$ & $\mathrm{Nb}$ & \\
\hline \multirow{2}{*}{ USLD 1} & M & $30 \%$ & 46 & & & $13 \%$ & 80 & & & $46 \%$ & 90 & 216 \\
\hline & $M$ & $21 \%$ & 46 & & & $8 \%$ & 44 & & & $48 \%$ & 71 & 161 \\
\hline \multirow{2}{*}{ USLD 2} & M & $30 \%$ & 71 & & & $11 \%$ & 47 & & & $43 \%$ & 49 & 167 \\
\hline & A & $24 \%$ & 47 & & & $17 \%$ & 99 & $0,1 \%$ & 2 & $45 \%$ & 58 & 206 \\
\hline \multirow{2}{*}{ EHPAD public 2} & M & $15 \%$ & 34 & $5 \%$ & 1 & & & $12 \%$ & 1 & $58 \%$ & 31 & 67 \\
\hline & $A$ & $19 \%$ & 16 & $5 \%$ & 1 & $10 \%$ & 19 & $4 \%$ & 1 & $48 \%$ & 31 & 68 \\
\hline EHPAD public 3 & $M$ & $19 \%$ & 56 & $12 \%$ & 7 & $23 \%$ & 134 & $3 \%$ & 5 & $39 \%$ & 62 & 264 \\
\hline \multirow{2}{*}{ EHPAD public 4} & $M$ & $17 \%$ & 57 & $20 \%$ & 23 & $26 \%$ & 65 & & & $18 \%$ & 73 & 218 \\
\hline & A & $25 \%$ & 44 & $4 \%$ & 9 & $34 \%$ & 73 & & & $27 \%$ & 27 & 153 \\
\hline $\begin{array}{r}\text { EHPAD Privé } \\
\text { non lucratif } 1\end{array}$ & J & $19 \%$ & 56 & $18 \%$ & 29 & $21 \%$ & 71 & & & $42 \%$ & 112 & 268 \\
\hline $\begin{array}{r}\text { EHPAD Privé } \\
\text { non lucratif } 3\end{array}$ & J & $22 \%$ & 93 & $23 \%$ & 7 & $9 \%$ & 79 & $0,1 \%$ & 1 & $42 \%$ & 52 & 232 \\
\hline \multicolumn{2}{|c|}{ Moyenne des 11 IDE } & $21,9 \%$ & 51 & $7,9 \%$ & 7 & $15,6 \%$ & 65 & $1,7 \%$ & 1 & $41,5 \%$ & 60 & 184 \\
\hline & & \multicolumn{2}{|c|}{$\begin{array}{c}\text { Chambres et leurs } \\
\text { sanitaire }\end{array}$} & \multicolumn{2}{|c|}{$\begin{array}{l}\text { Salon - salle à } \\
\text { manger }\end{array}$} & \multicolumn{2}{|c|}{ Couloirs escaliers } & \multicolumn{2}{|c|}{ Office } & \multicolumn{2}{|c|}{ Poste infirmier } & $\begin{array}{l}\text { Nombre total } \\
\text { de change ents }\end{array}$ \\
\hline $\begin{array}{c}\text { Aides- } \\
\text { soignants }\end{array}$ & horaire & $\%$ & $\mathrm{Nb}$ & $\%$ & $\mathrm{Nb}$ & $\%$ & $\mathrm{Nb}$ & $\%$ & $\mathrm{Nb}$ & $\%$ & $\mathrm{Nb}$ & de lieux \\
\hline \multirow{2}{*}{ USLD 1} & $M$ & $64 \%$ & 66 & $21 \%$ & 7 & $10 \%$ & 26 & & & $8 \%$ & 10 & 80 \\
\hline & $\mathrm{A}$ & $42 \%$ & 116 & & & $28 \%$ & 80 & & & $14 \%$ & 41 & 125 \\
\hline \multirow{2}{*}{ USLD 2} & $M$ & $40 \%$ & 31 & & & $11 \%$ & 33 & $1 \%$ & 2 & $14 \%$ & & 123 \\
\hline & $\mathrm{A}$ & $24 \%$ & 58 & & & $10 \%$ & 51 & $2 \%$ & 6 & $5 \%$ & & 171 \\
\hline \multirow{3}{*}{ EHPAD public 1} & $M$ & $41 \%$ & 38 & $6 \%$ & 6 & $13 \%$ & 34 & & & $8 \%$ & 2 & 149 \\
\hline & $M$ & $44 \%$ & 46 & $22 \%$ & 8 & $14 \%$ & 71 & & & & & 167 \\
\hline & A & $36 \%$ & 62 & $19 \%$ & 21 & $10 \%$ & 30 & & & $18 \%$ & 10 & 109 \\
\hline EHPAD publis? & $M$ & $46 \%$ & 55 & $11 \%$ & 19 & $15 \%$ & 75 & $16 \%$ & 14 & $7 \%$ & 8 & 382 \\
\hline EחPAD PUDIIC 2 & A & $68 \%$ & 100 & $0,5 \%$ & 3 & $6 \%$ & 31 & $14 \%$ & 5 & $3 \%$ & 10 & 303 \\
\hline E. public & $M$ & $40 \%$ & 65 & $7 \%$ & 9 & $13 \%$ & 82 & $26 \%$ & 10 & $0,9 \%$ & 1 & 121 \\
\hline 3 USLD 1 & $M$ & $64 \%$ & 66 & $21 \%$ & 7 & $10 \%$ & 26 & & & $8 \%$ & 10 & 93 \\
\hline & $M$ & $42 \%$ & 199 & $30 \%$ & 71 & $13 \%$ & 99 & & & $4 \%$ & 13 & 76 \\
\hline EHPAD public 4 & $M$ & $45 \%$ & 161 & $23 \%$ & 46 & $22 \%$ & 92 & & & $8 \%$ & 4 & 99 \\
\hline & A & $52 \%$ & 63 & $24 \%$ & 6 & $12 \%$ & 47 & & & $3 \%$ & 5 & 65 \\
\hline EHPA D public 5 & A & $28 \%$ & 21 & $6 \%$ & 29 & $48 \%$ & 31 & $8 \%$ & 11 & $0,1 \%$ & 1 & 68 \\
\hline LחFAD PUDIIC & $M$ & $45 \%$ & 31 & $1 \%$ & 3 & $11 \%$ & 35 & $5 \%$ & 6 & $3 \%$ & 1 & 247 \\
\hline E. Privé non & $\mathrm{J}$ & $45 \%$ & 47 & $11 \%$ & 8 & $12 \%$ & 42 & & & $8 \%$ & 2 & 132 \\
\hline lucratif 1 & $A$ & $29 \%$ & 14 & & & $45 \%$ & 48 & $13 \%$ & 3 & & & 92 \\
\hline & Md & $66 \%$ & 28 & & & $15 \%$ & 40 & & & & & 144 \\
\hline & Md & $64 \%$ & 135 & $0,8 \%$ & 5 & $18 \%$ & 107 & & & & & 48 \\
\hline EHPAD Privé & Md & $66 \%$ & 84 & $5 \%$ & 1 & $16 \%$ & 42 & $0,6 \%$ & 4 & $4 \%$ & 1 & 133 \\
\hline & $\mathrm{Ad}$ & $27 \%$ & 20 & $8 \%$ & 2 & $18 \%$ & 68 & & & $16 \%$ & 2 & 67 \\
\hline & $\mathrm{Ad}$ & $38 \%$ & 84 & & & $15 \%$ & 52 & $2 \%$ & 8 & & & 310 \\
\hline & $\mathrm{J}$ & $26 \%$ & 25 & & & $4 \%$ & 11 & $6 \%$ & 12 & & & 80 \\
\hline EHPAD Privé & $\mathrm{J}$ & $55 \%$ & 57 & $48 \%$ & 22 & $12 \%$ & 43 & $0,8 \%$ & 3 & $9 \%$ & 8 & 125 \\
\hline non lucratif 3 & $\mathrm{~A}$ & $28 \%$ & 19 & $13 \%$ & 16 & $19 \%$ & 30 & & & $16 \%$ & 2 & 123 \\
\hline & $\mathrm{J}$ & $50 \%$ & 143 & $17 \%$ & 4 & $17 \%$ & 127 & $0,2 \%$ & 2 & $5 \%$ & 34 & 171 \\
\hline Moyenne des 26 & & $44,3 \%$ & 58 & $10,5 \%$ & 11 & $16,4 \%$ & 47 & $3,6 \%$ & 3 & $5,9 \%$ & 4 & 122 \\
\hline
\end{tabular}

Légende $: \mathrm{M}=$ matin en général 7h50; $\mathrm{A}=$ après-midi; en général 7h50; $\mathrm{J}=$ journée; $\mathrm{d}=$ demi poste d'un horaire

Tableau 5. Temps passé dans les différents lieux au cours d'une journée de travail par les infirmiers et aides soignants de divers services de gérontologie (nombre de séjours réalisés et pourcentage du temps total de travail dans chaque catégorie de lieux)

Table 5. Time spent in different locations by registered nurses and nursing assistants working in geriatric care. (Number of periods for each location and percentage of total working time spent there.) 


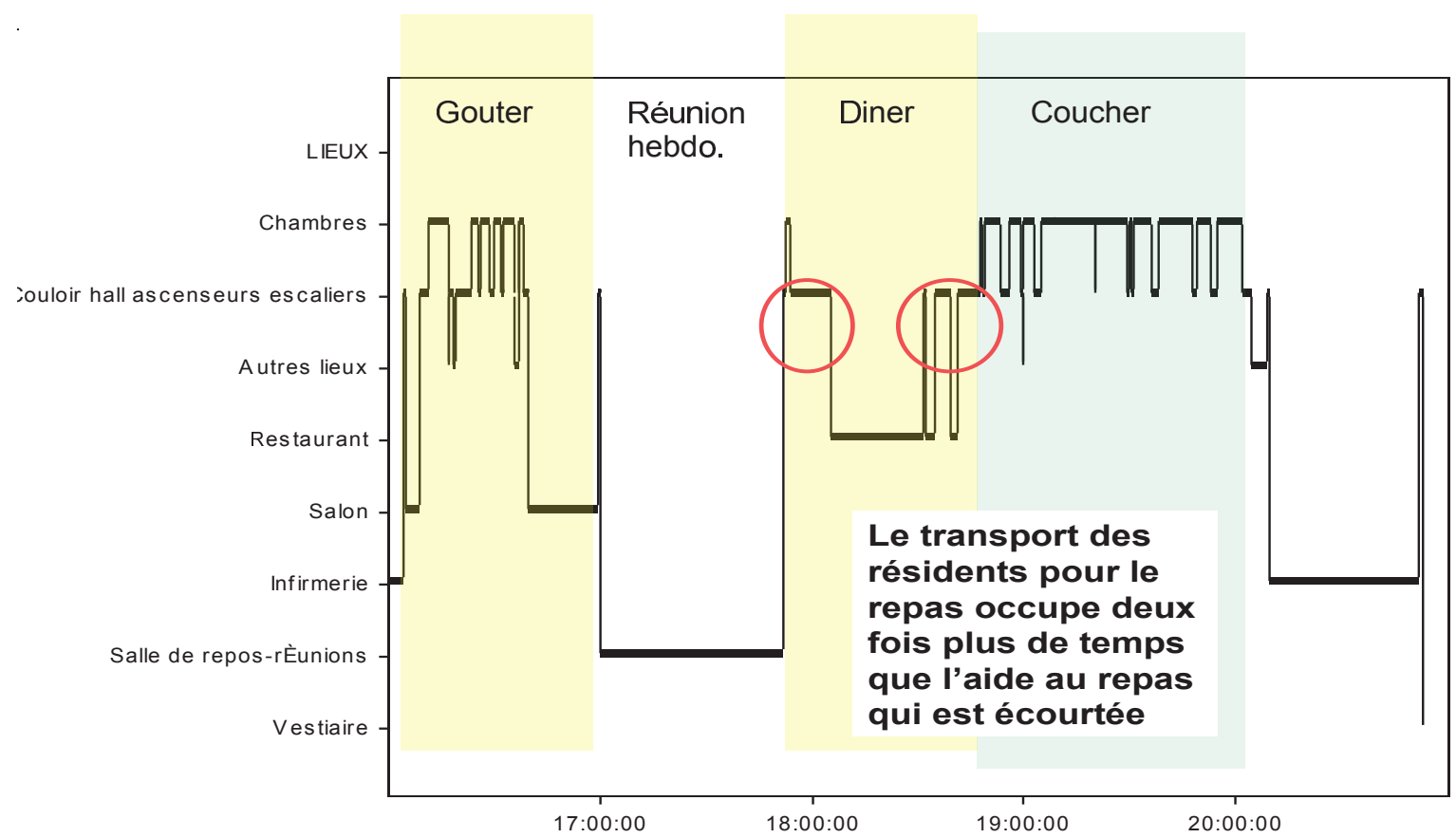

Figure 1. Déroulement chronologique des lieux de travail d'un agent de maison de retraite selon les grandes périodes d'activité

Figure 1. Work locations, in chronological order, of a nursing assistant working in a nursing home, and the main types of activity.

Note: Sur ce graphe réalisé avec le logiciel Kronos (Actogram Kronos @ élaboré par Kerguelen, 2008), les différentes chambres ne sont pas distinguées.

Note: On this graph, edited with KRONOS software, the different locations are on the same line. It shows that the time devoted to moving patients to the dining room by lift is twice as long as the time devoted to helping them eat. 